Supporting Information for

\title{
Identifying Toxicologically Significant Compounds in Urban Wildfire Ash using in Vitro Bioassays and High Resolution Mass Spectrometry
}

Thomas M. Young ${ }^{1,2}$, Gabrielle P. Black ${ }^{1}$, Luann Wong ${ }^{1}$, Clayton S. Bloszies ${ }^{2,3}$, Oliver Fiehn ${ }^{2,3}$, Guochun $\mathrm{He}^{4}$, Michael S. Denison ${ }^{4}$, Christoph F.A. Vogel ${ }^{5,}$ Blythe Durbin-Johnson ${ }^{6}$

${ }^{1}$ Department of Civil \& Environmental Engineering, ${ }^{2}$ Agricultural and Environmental Chemistry, ${ }^{3}$ West Coast Metabolomics Center, ${ }^{4}$ Department of Environmental Toxicology, ${ }^{5}$ Center for Health and the Environment, and ${ }^{6}$ Division of Biostatistics, School of Medicine

University of California, Davis

\section{List of Supporting Information Tables}

(Tables S1-S13 can be found in the supporting information Excel file)

Table S1. Sampling Location GPS

Table S2. LC Internal Standards

Table S3. Retention Index Calculation

Table S4. MS-DIAL GC Parameters

Table S5. GC Workflow Testing

Table S6. MS-DIAL LC Parameters

Table S7. LC Workflow Testing

Table S8. GC Target Results

Table S9. GC EI Alignment Summary

Table S10. LC ESI+ MS1 Alignment Summary

Table S11. LC ESI- MS1 Alignment Summary

Table S12. LC ESI+ MS2 Summary

Table S13. LC ESI- MS2 Summary

Table S14. Statistically significant $(\mathrm{p}<0.05)$ positive $(+)$ or negative (-) Spearman's rank-order correlation coefficients between aligned GC-EI molecular features and bioassay responses. 
Table S15. Statistically significant $(\mathrm{p}<0.05)$ Spearman rank correlation coefficients between aligned LCESI positive molecular features and bioassay responses.

Table S16. Statistically significant $(\mathrm{p}<0.05)$ Spearman rank correlation coefficients between aligned LCESI negative molecular features and bioassay responses.

Table S17. CALUX AhR Results for Nontarget GC-EI Features Selected for their Relationship to AhR Activity in Wildfire Ash Extracts

Table S18. ER Antagonism Test Results

Table S19. AR Antagonism Test Results

\section{List of Supporting Information Figures}

Figure S1. Effect of wildfire ash samples from Tubbs fire locations on cell viability. U937 derived macrophages were treated with extracts from ash samples for $24 \mathrm{~h}$ and cell viability was tested via trypan blue exclusion test.

Figure S2. Comparison of peak heights for confirmed polycyclic aromatic hydrocarbon standards from Agilent Quantitative Analysis B.09 with the peak heights of the corresponding aligned and identified features from MS-DIAL

Figure S3. Tentative identification, molecular structure, and mass spectra of GC-EI features listed in Table S14

Figure S4. MS-FINDER annotations for selected LC-ESI+ features listed in Table S15 including proposed molecular structures and comparison of experimental targeted MS/MS spectra and in silico MS/MS spectra

Figure S5. MS-FINDER annotations for selected LC-ESI- features listed in Table S16 including proposed molecular structures and comparison of experimental targeted MS/MS spectra and in silico MS/MS spectra 
Nontarget Workflow Parameter Selection and Performance. GC compounds were identified using the NIST17 library with equal weight assigned to mass spectral and retention index matching. A quadratic retention time-retention index relationship was developed for a list of 28 alkanes and aromatic compounds with standard nonpolar retention indexes between 800 and 3100 (NIST17) and the fitted parameters were used to develop a retention index dictionary for use with MS-DIAL (Table S3). GC alignment parameters were chosen to provide good compound isolation and identification on the alignment of a multilevel mixture (0.1-1000 ng/L) of 98 standard compounds that included normal alkanes, substituted benzenes, polycyclic aromatic hydrocarbons, and diverse organic compounds containing chlorine, sulfur, nitrogen, and silicon. The selected parameters (Table S4) yielded the correct molecular structure as the top hit for $61 \%$ of the compounds, produced a structural assignment that was an isomer of the correct compound for $22 \%$, produced an incorrect structure/molecular formula for $11 \%$, and failed to isolate the peak for 5\% (Table S5). Many of the isomeric assignments were to compounds with closely related structures (i.e., 1,3-dichlorobenzene instead of 1,4-dichlorobenzene) and the correct assignment was usually the second or third best hit, with the two isomers having very similar EI and RI match scores. Missed peaks typically coeluted with other, correctly identified peaks and could not be resolved with any of the tested alignment parameters. MS-DIAL parameters for LC data were developed to obtain good alignment and identification of a multilevel pesticide mixture containing 23 compounds using data independent (all ions) collision induced dissociation (Table S6). These parameters allowed $95 \%$ of the compounds to be correctly identified against a merged database containing all entries with MS2 spectra in the Agilent Water Contaminants, Pesticides, and Forensics/Toxicology libraries (Table S7). In $20 \%$ of the cases, however, the identification was based only on the MS1 accurate mass and did not include confirmation with an MS2 fragment ion; MS2 spectra for the compounds lacking MS2 confirmation typically feature relatively low fragment abundance and fragments were presumably 
missed as a result. For one compound (diuron, $5 \%$ of the total), the molecular feature was not correctly identified. 
Table S14. Statistically significant $(\mathrm{p}<0.05)$ positive $(+)$ or negative (-) Spearman's rank-order correlation coefficients between aligned GC-EI molecular features and bioassay responses. Also indicated are features selected as significant by the elastic net regression procedure $\left({ }^{*}\right)$ and compounds listed as AhR or ER activators in TOX21 testing, in an in-house database, or in targeted follow-up testing reported in Table S17 (A). A list of all aligned features is available in Table S9. Mass spectra for these 78 compounds, best match library spectra, and tentative molecular structure assignments are also provided in the Supporting Information (Figure S2).

\begin{tabular}{|c|c|c|c|c|c|c|c|}
\hline $\begin{array}{l}\text { Align } \\
\text { ID }\end{array}$ & Tentative Identification & Cyp1A1 & IL-8 & $\mathrm{COX}-2$ & AhR & ER & $\mathrm{AR}$ \\
\hline 23 & Pentane, 2,2,4-trimethyl- & - & - & & & & \\
\hline 30 & Pyridine & & & & & & + \\
\hline 38 & Heptane & - & & & & & \\
\hline 43 & $\begin{array}{l}\text { Oxirane, 2,3-bis(1-methylethyl)-, } \\
\text { trans- }\end{array}$ & & & - & - & & + \\
\hline 84 & Cyclopentane, 1-ethyl-3-methyl- & & & & & & + \\
\hline 86 & Trisiloxane, 1,1,3,3,5,5-hexamethyl- & - & & & & & \\
\hline 91 & Cyclopentane, (1-methylethyl)- & & & & - & & \\
\hline 103 & 2-Methyl-1,2-propanediamine & - & - & & & & \\
\hline 105 & 2-Chloro-3-methoxypropionamidine & - & & & & & \\
\hline 117 & 1-Butanol, 3-methyl-, acetate & - & & & & $+\mathbf{A}$ & + \\
\hline 118 & Pentane, 2,2,3,3-tetramethyl- & & & & & + & \\
\hline 122 & Thiophene, 2,5-dimethyl- & - & - & & & & \\
\hline 131 & Bicyclo[3.2.1]octane & & & & + & & \\
\hline 133 & $\begin{array}{l}\text { trans-2-Methylcyclohexanol, } \\
\text { heptafluorobutyrate }\end{array}$ & - & - & & & & \\
\hline 141 & 1-Octene, 3,7-dimethyl- & & & + & & & \\
\hline 144 & 2-Acetyl-1-pyrroline & & & & & & - \\
\hline 186 & 2-Heptanone, 6-methyl- & & & + & + & & \\
\hline 193 & Phenol & + & & & & - & \\
\hline 211 & Benzene, 1,4-dichloro- & - & - & & & & \\
\hline 216 & 1-Hexanol, 2-ethyl- & & & & & + & \\
\hline 221 & Benzaldehyde, 2-hydroxy- & + & + & & & $-\mathrm{A}$ & - \\
\hline 226 & Phenol, 2-methyl- & + & + & & & - & - \\
\hline 228 & Bis(2-chloroisopropyl) ether & + & + & + & & & - \\
\hline 231 & Nonane, 4,5-dimethyl- & & & & & + & \\
\hline 237 & Phenol, 3-methyl- & + & + & & & & - \\
\hline
\end{tabular}




\begin{tabular}{|c|c|c|c|c|c|c|c|}
\hline $\begin{array}{l}\text { Align } \\
\text { ID }\end{array}$ & Tentative Identification & Cyp1A1 & IL-8 & $\mathrm{COX}-2$ & AhR & ER & AR \\
\hline 250 & Phorone & & & & & & + \\
\hline 251 & Decane, 2,6,8-trimethyl- & + & + & + & & & - \\
\hline 255 & Phorone & - & & & & & \\
\hline 266 & $\begin{array}{l}\text { 2(5H)-Furanone, 5-(2-methyl-2- } \\
\text { propenyl)- }\end{array}$ & - & & & & & \\
\hline 268 & Phenol, 3,5-dimethyl- & + & + & & & - & \\
\hline 276 & 2-Methylisoborneol & & & & & & - \\
\hline 294 & 3-Ethylphenol, TMS derivative & . & + & & & & \\
\hline 296 & Benzofuran, 4,7-dimethyl- & & & & & - & \\
\hline 305 & Quinoline & $+*$ & $*$ & & & & \\
\hline 315 & Nonanoic acid & & $*$ & & & & \\
\hline 317 & $\begin{array}{l}\text { Benzeneethanol, .beta.-ethenyl- } \\
\text {.alpha.-methyl- }\end{array}$ & + & + & & & & \\
\hline 320 & Dodecane, 2,6,11-trimethyl- & & & & & + & \\
\hline 342 & Tridecane, 4-methyl- & + & & & + & & \\
\hline 343 & Benzenemethanol, 4-amino- & + & + & + & & & - \\
\hline 346 & Phenol, 2,3,6-trichloro- & + & + & & & & \\
\hline 349 & 4-Acetoxybenzaldehyde & + & $+*$ & + & $+\mathbf{A}$ & & - \\
\hline 351 & Benzene, heptyl- & $+*$ & & & & & \\
\hline 353 & Naphthalene, 1-chloro- & & & & & $-\mathrm{A}$ & \\
\hline 370 & o-Nitroaniline & & & & & - & \\
\hline 399 & Benzene, pentachloro- & & & & $+\mathbf{A}$ & & \\
\hline 411 & Benzo[b]thiophene, 2,3-diethyl- & & & + & & & \\
\hline 412 & Phenol, 2,3,4,6-tetrachloro- & & & & & - & \\
\hline 416 & Fluorene & + & + & & & & \\
\hline 425 & 2-(Methylmercapto)benzothiazole & & $*$ & & & & \\
\hline 430 & Benzophenone & & & + & & & \\
\hline 431 & $\begin{array}{l}\text { 1-[(4- } \\
\text { Ethoxyphenyl)methoxy]guanidine }\end{array}$ & + & & & & & \\
\hline 434 & $\begin{array}{l}\text { 2(3H)-benzothiazolone, 3-(1- } \\
\text { methylethyl)- }\end{array}$ & + & + & + & + & & - \\
\hline 454 & 9H-Fluoren-9-one & + & $+*$ & & & & \\
\hline 461 & Phenol, pentachloro- & & & & $+* A$ & & \\
\hline
\end{tabular}




\begin{tabular}{|c|c|c|c|c|c|c|c|}
\hline $\begin{array}{l}\text { Align } \\
\text { ID }\end{array}$ & Tentative Identification & Cyp1A1 & IL-8 & $\mathrm{COX}-2$ & AhR & ER & AR \\
\hline 465 & Phenanthrene & + & + & & & & \\
\hline 469 & Anthracene & $+*$ & $+*$ & $+*$ & $+^{*} \mathrm{~A}$ & & - \\
\hline 489 & Phenanthrene, 9-methyl- & $+*$ & $+*$ & $+*$ & & & - \\
\hline 497 & $\begin{array}{l}\text { Phthalic acid, isobutyl 2-methylallyl } \\
\text { ester }\end{array}$ & & + & + & & & \\
\hline 502 & Naphthalene, 2-phenyl- & + & + & + & $+\mathrm{A}$ & & \\
\hline 508 & $\begin{array}{l}\text { 1,3,5-Triazine-2,4-diamine, } 6- \\
\text { phenyl- }\end{array}$ & & & & - & & \\
\hline 514 & Fluoranthene & $+*$ & + & + & $+\mathbf{A}$ & $\mathbf{A}$ & - \\
\hline 515 & $\begin{array}{l}\text { Phenol, 2-[(4- } \\
\text { hydroxyphenyl)methyl]- }\end{array}$ & & + & & & & \\
\hline 517 & Pyrene & $+*$ & + & + & $+* \mathbf{A}$ & & \\
\hline 518 & $\begin{array}{l}\text { 1,2-Ethanediamine, N,N'-dimethyl- } \\
\text { N,N'-bis(phenylmethyl)- }\end{array}$ & & & + & & & \\
\hline 523 & Unknown & & + & & & & \\
\hline 526 & Quinoline, 2-(2-phenylethenyl)- & $+^{*}$ & + & $+*$ & $+\mathbf{A}$ & & \\
\hline 533 & Retene & & $*$ & & & - & \\
\hline 541 & Benzyl butyl phthalate & + & + & & & $\mathbf{A}$ & \\
\hline 542 & 1,3,7,9-Tetrachlorodibenzo-p-dioxin & $*$ & $*$ & & & & \\
\hline 550 & Unknown & & + & & & & \\
\hline 554 & Benz(a)anthracene & $+*$ & $+*$ & $+*$ & $+\mathbf{A}$ & $\mathbf{A}$ & - \\
\hline 555 & Chrysene & $+*$ & $+*$ & $+*$ & $+* \mathbf{A}$ & & \\
\hline 573 & Unknown & $+*$ & $+*$ & & & & - \\
\hline 574 & Benzo[b]fluoranthene & + & $+*$ & + & + & & \\
\hline 575 & Benzo[b]fluoranthene & $+*$ & $+*$ & * & & & \\
\hline 579 & 1,1':2',1"-Terphenyl, 4'-phenyl- & + & + & + & $+* \mathbf{A}$ & & \\
\hline 583 & Dibenz(a,h)anthracene & $*$ & $+*$ & + & $+\mathbf{A}$ & & \\
\hline 591 & Unknown & & + & & & & \\
\hline
\end{tabular}


Table S15. Statistically significant $(p<0.05)$ Spearman rank order correlation coefficients between aligned LC-ESI positive molecular features and bioassay responses.

\begin{tabular}{|l|c|c|c|c|c|c|c|}
\hline $\begin{array}{l}\text { Align } \\
\text { ID }\end{array}$ & Cyp1A1 & IL-8 & COX-2 & AhR & ER & AR & $\begin{array}{c}\text { MS/MS } \\
\text { Acquired }\end{array}$ \\
\hline 0 & - & & & & & & \\
\hline 16 & & & - & & & & \\
\hline 51 & - & & & & & & \\
\hline 59 & & & & & + & & \\
\hline 60 & - & & & & & & \\
\hline 95 & + & + & & & & & \\
\hline 110 & - & & & & & & \\
\hline 114 & - & & & & & & \\
\hline 119 & & + & & & & - & \\
\hline 127 & - & & & & & & \\
\hline 131 & + & + & & & & & \\
\hline 143 & & + & & & & & \\
\hline 144 & & + & & & & & \\
\hline 151 & - & - & & - & & + & \\
\hline 157 & + & + & & & & & X \\
\hline 159 & & + & & & & - & \\
\hline 163 & + & + & & & & & \\
\hline 165 & + & + & & + & & & \\
\hline 171 & & & & + & & & \\
\hline 172 & & + & & & & - & \\
\hline 173 & + & + & & & & - & \\
\hline 186 & - & & & & & & \\
\hline 188 & - & & & & & & \\
\hline 197 & - & - & & & & + & \\
\hline 207 & + & + & & & & - & X \\
\hline 215 & & + & & & & - & \\
\hline 217 & & & & & + & & \\
\hline 221 & + & + & + & & & - & \\
\hline 243 & - & - & & - & & + & X \\
\hline 254 & & + & & & & - & \\
\hline 256 & & & & & - & & \\
\hline 262 & & & & & - & - & X \\
\hline 265 & & + & & & & - & \\
\hline 283 & + & & & & & & \\
\hline 290 & & & + & & & & \\
\hline 309 & & + & & & & - & \\
\hline & & & & & & & \\
\hline
\end{tabular}




\begin{tabular}{|c|c|c|c|c|c|c|c|}
\hline $\begin{array}{l}\text { Align } \\
\text { ID }\end{array}$ & Cyp1A1 & IL-8 & COX-2 & AhR & ER & AR & $\begin{array}{c}\text { MS/MS } \\
\text { Acquired }\end{array}$ \\
\hline 310 & & & & & & - & \\
\hline 317 & & + & & & & - & \\
\hline 331 & - & & & & & & \\
\hline 343 & - & & & & & & \\
\hline 368 & & - & & & & & \\
\hline 377 & - & & & & & & \\
\hline 385 & + & + & & & & & \\
\hline 417 & & + & & & & - & \\
\hline 421 & & + & & & & - & \\
\hline 423 & & & & & & - & \\
\hline 441 & + & + & + & + & & - & \\
\hline 443 & & & & + & & & \\
\hline 458 & + & + & & & & & $\mathrm{X}$ \\
\hline 465 & - & & & & & & \\
\hline 468 & - & & & & & & \\
\hline 475 & & & + & & - & & \\
\hline 483 & + & + & & & & & \\
\hline 485 & + & + & & & & . & \\
\hline 488 & + & + & & & & - & \\
\hline 506 & & & & + & & & \\
\hline 546 & & + & & & & - & \\
\hline 552 & + & + & & & & - & $\mathrm{X}$ \\
\hline 556 & & + & & & & - & \\
\hline 562 & & + & & & & & \\
\hline 563 & - & & & & & & \\
\hline 579 & + & + & + & + & & 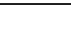 & \\
\hline 581 & & & & & - & - & \\
\hline 628 & & & & & + & & \\
\hline 649 & + & + & & & & & \\
\hline 673 & & + & & & & - & \\
\hline 683 & + & + & & & & - & \\
\hline 702 & & & & & & - & \\
\hline 711 & + & + & + & & & - & \\
\hline 713 & & & & & $*$ & & \\
\hline 715 & + & + & + & + & & & \\
\hline 727 & & & & & & - & \\
\hline 729 & + & & & & & & \\
\hline 736 & + & + & & & & & \\
\hline
\end{tabular}




\begin{tabular}{|c|c|c|c|c|c|c|c|}
\hline $\begin{array}{l}\text { Align } \\
\text { ID }\end{array}$ & Cyp1A1 & IL-8 & COX-2 & AhR & ER & AR & $\begin{array}{c}\text { MS/MS } \\
\text { Acquired }\end{array}$ \\
\hline 737 & + & + & & & & & \\
\hline 744 & & - & & - & & & \\
\hline 753 & + & + & & & & - & $X$ \\
\hline 757 & + & + & & & - & - & $\mathrm{X}$ \\
\hline 758 & & & & & & - & \\
\hline 783 & - & & & - & + & + & \\
\hline 784 & & & & & - & & \\
\hline 786 & & & & & + & & \\
\hline 789 & & + & & & & - & \\
\hline 791 & & & & - & & & \\
\hline 795 & & + & & & & - & \\
\hline 840 & & & & & - & & \\
\hline 851 & & & & - & & & \\
\hline 862 & . & & & + & & & \\
\hline 896 & + & + & + & + & & & \\
\hline 901 & & & & + & & & \\
\hline 902 & & 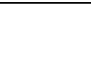 & & + & & & \\
\hline 923 & 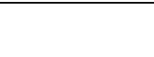 & - & & - & & 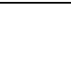 & \\
\hline 926 & + & + & + & & & - & \\
\hline 940 & & & & & & - & \\
\hline 943 & . & + & & & & - & \\
\hline 944 & + & + & & & & & $X$ \\
\hline 964 & & & & & & $*$ & $X$ \\
\hline 969 & - & & & & & & \\
\hline 987 & . & & & & - & & \\
\hline 1010 & & + & & & . & - & $X$ \\
\hline 1020 & & & & & - & & \\
\hline 1021 & & & & & - & & \\
\hline 1024 & + & $+*$ & & & & - & $\mathrm{X}$ \\
\hline 1026 & & & - & & & $+*$ & \\
\hline 1030 & - & & & & & & \\
\hline 1035 & & & & & & - & \\
\hline 1043 & & & & & & $*$ & \\
\hline 1054 & & & & & $*$ & & $X$ \\
\hline 1057 & - & & - & & & & \\
\hline 1076 & + & & & + & & & \\
\hline 1077 & & & & & - & & \\
\hline 1092 & & & & & - & & \\
\hline
\end{tabular}




\begin{tabular}{|c|c|c|c|c|c|c|c|}
\hline $\begin{array}{l}\text { Align } \\
\text { ID }\end{array}$ & Cyp1A1 & IL-8 & COX-2 & AhR & ER & AR & $\begin{array}{c}\text { MS/MS } \\
\text { Acquired }\end{array}$ \\
\hline 1094 & & & & & - & & \\
\hline 1104 & + & $+*$ & & & & - & \\
\hline 1122 & + & + & & + & & - & \\
\hline 1143 & & & & & - & & \\
\hline 1160 & & + & & & & - & \\
\hline 1176 & & & & - & & & \\
\hline 1180 & & & & + & & & \\
\hline 1193 & & & & & - & & \\
\hline 1206 & + & + & & & & & \\
\hline 1209 & & & & & - & & \\
\hline 1210 & & & & & - & - & \\
\hline 1219 & & & & - & & & \\
\hline 1226 & & & - & & & & \\
\hline 1234 & - & - & & - & . & + & \\
\hline 1237 & & & & & & $*$ & $\mathrm{X}$ \\
\hline 1238 & & & & & & $*$ & $\mathrm{X}$ \\
\hline 1241 & & & & & - & & \\
\hline 1243 & + & & & & & & \\
\hline 1248 & & & & & - & & \\
\hline 1249 & - & - & & - & & + & \\
\hline 1269 & & & & & & - & \\
\hline 1279 & & & + & + & & . & \\
\hline 1296 & & + & & & & - & $X$ \\
\hline 1306 & + & + & & + & & - & \\
\hline 1310 & & & & & & - & \\
\hline 1316 & + & & & & & & \\
\hline 1326 & & + & & & & - & \\
\hline 1330 & & & & & - & & \\
\hline 1331 & & & & & & - & \\
\hline 1338 & & + & & & - & - & \\
\hline 1346 & & & & & - & & \\
\hline 1350 & & & & - & & & \\
\hline 1351 & & & & + & & & \\
\hline 1361 & & & & + & & & \\
\hline 1385 & & & & & - & - & \\
\hline 1390 & & - & & & & & \\
\hline 1405 & & & & & - & & \\
\hline 1406 & & & - & & & $*$ & \\
\hline
\end{tabular}




\begin{tabular}{|c|c|c|c|c|c|c|c|}
\hline $\begin{array}{l}\text { Align } \\
\text { ID }\end{array}$ & Cyp1A1 & IL-8 & COX-2 & AhR & ER & AR & $\begin{array}{c}\text { MS/MS } \\
\text { Acquired }\end{array}$ \\
\hline 1414 & & + & & & & - & X \\
\hline 1415 & & & + & + & & & \\
\hline 1422 & + & + & + & + & & - & $\mathrm{X}$ \\
\hline 1423 & & + & & & & - & \\
\hline 1443 & + & + & & & & - & \\
\hline 1456 & & & & - & & & \\
\hline 1484 & & & & - & & & \\
\hline 1501 & + & & & & & & \\
\hline 1502 & & & & & & $*$ & $\mathrm{X}$ \\
\hline 1512 & & + & & & & & \\
\hline 1519 & & & & & - & & \\
\hline 1547 & & & & - & & & \\
\hline 1563 & & & & - & & & \\
\hline 1611 & & & - & - & & $*$ & $\mathrm{X}$ \\
\hline 1637 & & & & + & & & \\
\hline 1645 & + & + & & & & & \\
\hline 1649 & & & & & & - & \\
\hline 1667 & & & & - & & & \\
\hline 1674 & & & & & & - & \\
\hline 1687 & & & & - & & & \\
\hline 1697 & + & + & & + & & & \\
\hline 1716 & & & & - & + & & \\
\hline 1725 & & & & & - & & \\
\hline 1727 & & & - & . & & & \\
\hline 1747 & - & & & . & & & \\
\hline 1769 & & & & - & & & \\
\hline 1777 & & & & - & & & \\
\hline 1778 & & & & & & - & \\
\hline 1792 & & & & + & & & \\
\hline 1808 & & & & & & $*$ & \\
\hline 1828 & & & & & & $*$ & \\
\hline 1845 & & & & & & - & \\
\hline 1867 & & & & + & & & \\
\hline 1877 & & & & - & & & \\
\hline 1882 & & & & + & & & \\
\hline 1903 & - & & & & & & \\
\hline 1912 & + & + & & & & - & \\
\hline 1915 & + & & & & & & \\
\hline
\end{tabular}




\begin{tabular}{|c|c|c|c|c|c|c|c|}
\hline $\begin{array}{l}\text { Align } \\
\text { ID }\end{array}$ & Cyp1A1 & IL-8 & COX-2 & AhR & ER & AR & $\begin{array}{c}\text { MS/MS } \\
\text { Acquired }\end{array}$ \\
\hline 1929 & & & & & & - & \\
\hline 1952 & & & - & & & & \\
\hline 1985 & & & & & $*$ & $*$ & $\mathrm{X}$ \\
\hline 1996 & & & & & & - & \\
\hline 2024 & & & - & & & & \\
\hline 2039 & + & + & & & - & & \\
\hline 2067 & & & & & & - & \\
\hline 2074 & + & & & & & & \\
\hline 2079 & + & & & & & - & \\
\hline 2100 & & + & & & & & \\
\hline 2130 & + & & & & & & \\
\hline 2151 & + & + & & & & - & \\
\hline 2177 & & & & & - & & \\
\hline 2199 & & - & & & & & \\
\hline 2213 & - & & & - & & & \\
\hline 2220 & + & + & + & + & & - & $\mathrm{X}$ \\
\hline 2233 & & & & + & & & \\
\hline 2245 & & & - & & & & \\
\hline 2286 & & + & & & & - & \\
\hline 2311 & & & & & - & & \\
\hline 2340 & + & & & & - & & \\
\hline 2346 & & + & & & & & \\
\hline 2370 & & & & & & - & \\
\hline 2372 & & & - & & . & & \\
\hline 2381 & & & & & - & & \\
\hline 2383 & & + & & & & & \\
\hline 2415 & & + & & & & & \\
\hline 2436 & & + & & & & & \\
\hline 2444 & + & & & & - & & $\mathrm{X}$ \\
\hline 2449 & & & & & - & & \\
\hline 2457 & & & & + & & & \\
\hline 2464 & & & & & & - & \\
\hline 2509 & - & & & & & & \\
\hline 2510 & & + & & & & & \\
\hline 2517 & & & & & & - & \\
\hline 2524 & & + & & & & & \\
\hline 2534 & & & - & & & & \\
\hline 2538 & & & - & & & & \\
\hline
\end{tabular}




\begin{tabular}{|c|c|c|c|c|c|c|c|}
\hline $\begin{array}{l}\text { Align } \\
\text { ID }\end{array}$ & Cyp1A1 & IL-8 & COX-2 & AhR & ER & AR & $\begin{array}{c}\text { MS/MS } \\
\text { Acquired }\end{array}$ \\
\hline 2540 & + & & & & & & \\
\hline 2552 & - & & & & & & \\
\hline 2553 & - & & & & & & \\
\hline 2571 & & & & + & & & \\
\hline 2604 & & & & & $*$ & & $\mathrm{X}$ \\
\hline 2608 & & & - & & & & $\mathrm{X}$ \\
\hline 2610 & & & & & - & & \\
\hline 2642 & & & & + & & & \\
\hline 2684 & & & & & & - & \\
\hline 2712 & & - & - & & & + & \\
\hline 2717 & & & - & & & & \\
\hline 2718 & & & & + & & & \\
\hline 2724 & & & - & & & & \\
\hline 2742 & & & & + & & & \\
\hline 2747 & & + & & & & & \\
\hline 2782 & & & & + & & & \\
\hline 2802 & & & & + & & & \\
\hline 2830 & & + & & & & & \\
\hline 2854 & & - & & & & & \\
\hline 2855 & + & + & & & & - & \\
\hline 2865 & & & - & & & & \\
\hline 2867 & & & - & & & & \\
\hline 2896 & & - & & & & & \\
\hline 2904 & & & 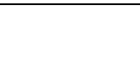 & & - & & \\
\hline 2906 & & & - & & & & \\
\hline 2926 & & & & & & $*$ & \\
\hline 2930 & & + & & & & & \\
\hline 2970 & & & & + & & & \\
\hline 2984 & & & & & - & & \\
\hline 2991 & & & - & & & & \\
\hline 2992 & & & - & & & & \\
\hline 3005 & & & & & + & & \\
\hline 3089 & & & - & & & & \\
\hline 3207 & - & & & & & & \\
\hline 3208 & & & & + & & & \\
\hline 3294 & & - & & & & & \\
\hline 3307 & & & + & & & & \\
\hline 3344 & & & & + & & & \\
\hline
\end{tabular}




\begin{tabular}{|c|c|c|c|c|c|c|c|}
\hline $\begin{array}{l}\text { Align } \\
\text { ID }\end{array}$ & Cyp1A1 & IL-8 & COX-2 & AhR & ER & AR & $\begin{array}{c}\text { MS/MS } \\
\text { Acquired }\end{array}$ \\
\hline 3444 & & & - & & & & \\
\hline 3454 & & & & & & $*$ & \\
\hline 3458 & & - & & & & & \\
\hline 3492 & & - & & & & & \\
\hline 3630 & & & - & & & & \\
\hline 3636 & & - & & & & & \\
\hline 3639 & & - & & & & & \\
\hline 3640 & & - & & & & & \\
\hline 3642 & & - & & & & & \\
\hline 3649 & - & . & & - & & & \\
\hline 3657 & & & - & & & & \\
\hline 3704 & & - & 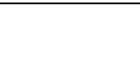 & & & & \\
\hline 3705 & & - & . & & & & \\
\hline 3726 & & & - & & & & \\
\hline 3731 & & & - & & & & \\
\hline 3774 & & & - & & & & \\
\hline 3782 & & & - & & & & \\
\hline 3788 & & & & & & - & \\
\hline 3815 & & & - & & & & \\
\hline 3852 & & & - & & & & \\
\hline 3854 & & + & . & & - & - & \\
\hline 3887 & & & - & & & & \\
\hline 3903 & & & - & & & & \\
\hline 3932 & - & - & & & & & \\
\hline 3979 & & & - & & & & \\
\hline
\end{tabular}


Table S16. Statistically significant $(p<0.05)$ Spearman rank order correlation coefficients between aligned LC-ESI negative molecular features and bioassay responses.

\begin{tabular}{|l|c|c|c|c|c|c|c|}
\hline $\begin{array}{l}\text { Align } \\
\text { ID }\end{array}$ & Cyp1A1 & IL-8 & COX-2 & AhR & ER & AR & $\begin{array}{c}\text { MS/MS } \\
\text { Acquired }\end{array}$ \\
\hline 10 & & + & & & & & \\
\hline 11 & & & & & & - & \\
\hline 18 & - & - & & - & & + & X \\
\hline 22 & - & & & & & & \\
\hline 38 & & & & & - & & \\
\hline 78 & - & - & & - & & + & X \\
\hline 80 & + & + & & & & & X \\
\hline 82 & & & & & & - & \\
\hline 106 & & & & & & - & \\
\hline 107 & & & & & & - & \\
\hline 109 & & & & & & - & \\
\hline 116 & + & + & & & & & \\
\hline 121 & & + & & & & & \\
\hline 134 & & & - & & & + & \\
\hline 143 & + & + & & & & - & X \\
\hline 156 & + & + & + & & - & - & X \\
\hline 157 & & & & & & - & \\
\hline 158 & & + & & & & - & \\
\hline 159 & & + & & & & & \\
\hline 160 & & + & & & & - & X \\
\hline 167 & & & & & & - & \\
\hline 179 & + & + & & & & & \\
\hline 180 & & - & & & & & \\
\hline 181 & + & + & & & & & \\
\hline 182 & & & & & - & & \\
\hline 184 & & & & & - & & \\
\hline 185 & + & + & & & & & X \\
\hline 189 & & & & & & - & \\
\hline 198 & + & + & & & & & \\
\hline 200 & & + & & & & & \\
\hline 207 & & & & & - & & \\
\hline 223 & + & + & & & & & \\
\hline 227 & + & + & & & & & \\
\hline 230 & + & + & & & & & \\
\hline 231 & + & + & & & & & \\
\hline 234 & + & + & & & & & \\
\hline
\end{tabular}




\begin{tabular}{|c|c|c|c|c|c|c|c|}
\hline $\begin{array}{l}\text { Align } \\
\text { ID }\end{array}$ & Cyp1A1 & IL-8 & COX-2 & AhR & ER & AR & $\begin{array}{c}\text { MS/MS } \\
\text { Acquired }\end{array}$ \\
\hline 235 & + & + & & & & & X \\
\hline 236 & + & + & & & & & \\
\hline 237 & & & & & & - & \\
\hline 238 & & & & & - & & \\
\hline 240 & & & & & - & & \\
\hline 245 & & & & & & - & \\
\hline 248 & & + & & & & - & \\
\hline 250 & + & + & + & + & & - & \\
\hline 254 & + & + & & & & & \\
\hline 255 & & & & & & - & \\
\hline 264 & & & & & & - & \\
\hline 265 & + & + & + & & - & - & $X$ \\
\hline 266 & & & & & & - & \\
\hline 268 & + & + & & & & & \\
\hline 273 & & + & & & & - & \\
\hline 277 & - & & & & & & \\
\hline 289 & + & + & & & & 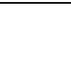 & $X$ \\
\hline 290 & & + & & & & - & \\
\hline 291 & - & + & & & & - & \\
\hline 292 & + & + & & & & . & \\
\hline 294 & + & + & & & & . & \\
\hline 295 & - & & & & & & \\
\hline 297 & & + & & & & - & \\
\hline 302 & & + & & & & . & \\
\hline 303 & & + & & & & 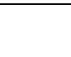 & \\
\hline 304 & & + & & & & - & \\
\hline 309 & . & + & & & & - & \\
\hline 314 & + & + & & & & 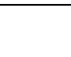 & \\
\hline 323 & & + & & & & - & \\
\hline 334 & + & + & & & & & \\
\hline 340 & & & & & & - & \\
\hline 353 & & + & & & & - & \\
\hline 371 & + & + & & & & & $\mathrm{X}$ \\
\hline 374 & & + & & & & - & \\
\hline 376 & + & + & & & & & \\
\hline 377 & & + & & & & - & \\
\hline 378 & & + & & & & - & \\
\hline 379 & & + & & & & - & \\
\hline
\end{tabular}




\begin{tabular}{|c|c|c|c|c|c|c|c|}
\hline $\begin{array}{l}\text { Align } \\
\text { ID }\end{array}$ & Cyp1A1 & IL-8 & COX-2 & AhR & ER & AR & $\begin{array}{c}\text { MS/MS } \\
\text { Acquired }\end{array}$ \\
\hline 381 & + & + & & & & & \\
\hline 382 & & + & & & & - & $\mathrm{X}$ \\
\hline 388 & & + & & & & - & \\
\hline 389 & & + & & & & & \\
\hline 393 & & + & & & & - & \\
\hline 400 & & + & & & & & \\
\hline 415 & & + & & & & - & $\mathrm{X}$ \\
\hline 419 & & + & & & & - & $\mathrm{X}$ \\
\hline 433 & & + & & & & - & \\
\hline 435 & & + & & & & - & \\
\hline 436 & & + & & & & - & \\
\hline 438 & + & + & & & & & $X$ \\
\hline 440 & & & & & & - & \\
\hline 442 & & & & - & + & & $\mathrm{X}$ \\
\hline 444 & & + & & & & & \\
\hline 448 & & & & & - & & \\
\hline 451 & + & + & & & & & $X$ \\
\hline 452 & + & + & & & & . & \\
\hline 458 & + & + & + & + & & . & \\
\hline 461 & & + & & & & - & $X$ \\
\hline 465 & & & & & & - & \\
\hline 468 & & + & & & & - & \\
\hline 469 & & + & & & & . & \\
\hline 470 & & + & & & & 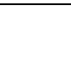 & \\
\hline 473 & & + & & & & - & \\
\hline 482 & & + & & & & - & $X$ \\
\hline 494 & & & & & - & . & \\
\hline 495 & & + & & & & - & \\
\hline 518 & & + & & & & - & \\
\hline 525 & & + & & & & - & $X$ \\
\hline 530 & + & + & + & & & - & $X$ \\
\hline 551 & & & - & & & & \\
\hline 552 & & & & & - & & \\
\hline 570 & - & & & & & & \\
\hline 571 & & + & & & & - & $\mathrm{X}$ \\
\hline 597 & & & & - & & & \\
\hline 617 & + & + & & & & - & $\mathrm{X}$ \\
\hline 642 & & + & & & & - & \\
\hline
\end{tabular}




\begin{tabular}{|c|c|c|c|c|c|c|c|}
\hline $\begin{array}{l}\text { Align } \\
\text { ID }\end{array}$ & Cyp1A1 & IL-8 & COX-2 & AhR & ER & AR & $\begin{array}{c}\text { MS/MS } \\
\text { Acquired }\end{array}$ \\
\hline 648 & & + & & & & - & X \\
\hline 650 & & + & & & & - & \\
\hline 651 & & + & & & & - & \\
\hline 652 & & + & & & & & \\
\hline 653 & + & + & & & - & - & $\mathrm{X}$ \\
\hline 654 & & + & & & & & \\
\hline 655 & + & + & + & & & - & $\mathrm{X}$ \\
\hline 668 & & + & & & & - & \\
\hline 726 & & & & + & & & \\
\hline 728 & & + & & & & & \\
\hline 730 & & + & & & & & \\
\hline 731 & & + & & & & & \\
\hline 747 & & + & + & & & - & $\mathrm{X}$ \\
\hline 763 & & + & & & & & \\
\hline 765 & & & & - & & & \\
\hline 784 & & & & & & - & \\
\hline 791 & & + & & & & - & $X$ \\
\hline 793 & & & & & & - & \\
\hline 798 & & 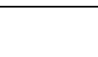 & & & & - & \\
\hline 805 & & + & + & & & - & \\
\hline 809 & & + & & & & - & $X$ \\
\hline 811 & + & + & + & & - & - & \\
\hline 814 & & + & & & & - & $X$ \\
\hline 817 & & & & & & - & \\
\hline 818 & + & + & + & & - & - & $\mathrm{X}$ \\
\hline 820 & + & + & & & & - & $\mathrm{X}$ \\
\hline 843 & & & & - & & & \\
\hline 854 & & + & & & & - & \\
\hline 865 & + & + & & & & - & \\
\hline 870 & & + & & & & - & $\mathrm{X}$ \\
\hline 871 & + & + & + & + & & - & \\
\hline 875 & + & + & & & & - & $\mathrm{X}$ \\
\hline 877 & & + & & & & & \\
\hline 882 & & + & & & & & \\
\hline 883 & + & + & & & & & \\
\hline 884 & & - & & & & & \\
\hline 889 & & + & & & & - & $\mathrm{X}$ \\
\hline 890 & & + & & & & - & $X$ \\
\hline
\end{tabular}




\begin{tabular}{|c|c|c|c|c|c|c|c|}
\hline $\begin{array}{l}\text { Align } \\
\text { ID }\end{array}$ & Cyp1A1 & IL-8 & COX-2 & AhR & ER & AR & $\begin{array}{c}\text { MS/MS } \\
\text { Acquired }\end{array}$ \\
\hline 891 & & + & & & & - & \\
\hline 920 & & + & & & & - & $X$ \\
\hline 938 & & & + & & & & \\
\hline 947 & + & & & & & - & \\
\hline 954 & & & - & & & + & $\mathrm{X}$ \\
\hline 961 & & & - & & & & \\
\hline 962 & & + & & & & - & \\
\hline 975 & & + & & & & & \\
\hline 984 & + & + & & & & - & $X$ \\
\hline 988 & & + & & & & - & $X$ \\
\hline 990 & & + & & & & - & \\
\hline 1000 & & + & & & & - & $X$ \\
\hline 1001 & & + & & & & - & $X$ \\
\hline 1009 & & + & & & & . & \\
\hline 1010 & & + & & & & - & $\mathrm{X}$ \\
\hline 1011 & & + & & & & - & \\
\hline 1012 & & + & & & - & - & $X$ \\
\hline 1024 & & & & & & - & \\
\hline 1025 & + & + & & & & - & $X$ \\
\hline 1029 & + & + & & & & - & $\mathrm{X}$ \\
\hline 1030 & & & & & & - & \\
\hline 1033 & & & & & - & & \\
\hline 1052 & & + & & & - & & \\
\hline 1067 & & + & & & & - & $X$ \\
\hline 1075 & + & + & & & & & $\mathrm{X}$ \\
\hline 1096 & + & + & & & & & \\
\hline 1097 & & & & & & - & \\
\hline 1107 & & & & & - & & \\
\hline 1111 & & & & + & & & \\
\hline 1131 & & & & & - & - & \\
\hline 1140 & & & & & & - & \\
\hline 1143 & & & & & & - & \\
\hline 1144 & & & & & & - & \\
\hline 1146 & + & + & & & - & - & $X$ \\
\hline 1148 & + & + & + & & - & - & $X$ \\
\hline 1149 & + & + & + & & - & - & \\
\hline 1151 & + & & & & & & \\
\hline 1164 & & + & & & & - & \\
\hline
\end{tabular}




\begin{tabular}{|c|c|c|c|c|c|c|c|}
\hline $\begin{array}{l}\text { Align } \\
\text { ID }\end{array}$ & Cyp1A1 & IL-8 & COX-2 & AhR & ER & AR & $\begin{array}{c}\text { MS/MS } \\
\text { Acquired }\end{array}$ \\
\hline 1165 & & + & & & & - & \\
\hline 1179 & & & & & - & - & \\
\hline 1181 & & + & & & & - & \\
\hline 1183 & & & & - & & & \\
\hline 1204 & & + & & & & - & \\
\hline 1239 & & & & + & & & \\
\hline 1255 & + & + & & & - & - & $X$ \\
\hline 1282 & + & & & & & & \\
\hline 1334 & & & & & & - & \\
\hline 1350 & & & & & & - & \\
\hline 1397 & & & & & & - & \\
\hline 1433 & & - & & & & & \\
\hline 1438 & + & + & & & & & \\
\hline 1441 & & + & & & & & \\
\hline 1466 & - & & & & & & \\
\hline 1469 & - & & & & & + & $X$ \\
\hline 1480 & & & & + & & & \\
\hline 1481 & & & & & + & & \\
\hline 1495 & & & & & - & & \\
\hline 1516 & & & + & + & & & \\
\hline 1536 & + & & & & & & \\
\hline 1547 & & & & + & & & \\
\hline 1548 & & & & + & & & \\
\hline 1562 & & & & + & & & \\
\hline 1569 & & & & + & & & \\
\hline 1580 & & & & + & & & \\
\hline 1585 & & & & + & & & \\
\hline 1592 & + & + & & & & & \\
\hline 1602 & & & + & & & & \\
\hline 1610 & & & - & & & & \\
\hline 1611 & & & & & + & + & \\
\hline 1660 & + & + & & & & & \\
\hline 1673 & & & & + & & & \\
\hline 1674 & & & & + & & & \\
\hline 1698 & & - & & & + & & \\
\hline
\end{tabular}


Table S17. CALUX AhR Results for Nontarget GC-EI Features Selected for their Relationship to AhR Activity in Wildfire Ash Extracts. Standard deviations are based on triplicate measurements.

\begin{tabular}{|l|l|l|l|l|}
\hline Compound & concentration & $\begin{array}{l}\text { \% of 1nM } \\
\text { TCDD }\end{array}$ & $\begin{array}{l}\text { Standard } \\
\text { deviation }\end{array}$ & $\begin{array}{l}\text { p-value for } \\
\text { difference from } \\
\text { DMSO control }\end{array}$ \\
\hline TCDD & $1 \mathrm{nM}$ & 100 & 15.99 & \\
\hline DMSO & blank & 0.46 & 0.54 & \\
\hline \multirow{5}{*}{ 4-Acetoxy-benzaldehyde } & $1 \mu \mathrm{M}$ & -0.04 & 0.24 & 0.912 \\
\cline { 2 - 5 } & $10 \mu \mathrm{M}$ & 0.57 & 0.60 & 0.0954 \\
\cline { 2 - 5 } & $100 \mu \mathrm{M}$ & $5.64^{*}$ & 0.92 & 0.00156 \\
\hline 2-phenyl-naphthalene & $1 \mu \mathrm{M}$ & 4.89 & 1.13 & 0.00566 \\
\cline { 2 - 5 } & $10 \mu \mathrm{M}$ & 18.81 & 0.97 & 0.000306 \\
\cline { 2 - 5 } & $100 \mu \mathrm{M}$ & 141.45 & 14.85 & 0.00347 \\
\hline 1,2,4-triphenyl benzene & $1 \mu \mathrm{M}$ & 11.69 & 2.93 & 0.0145 \\
\cline { 2 - 5 } & $10 \mu \mathrm{M}$ & 8.80 & 3.28 & 0.0314 \\
\cline { 2 - 5 } & $100 \mu \mathrm{M}$ & 7.29 & 1.48 & 0.00964 \\
\hline \multirow{3}{*}{ pentachlorobenzene } & $1 \mu \mathrm{M}$ & 2.00 & 0.15 & 0.0348 \\
\cline { 2 - 5 } & $10 \mu \mathrm{M}$ & 23.29 & 7.54 & 0.0375 \\
\cline { 2 - 5 } & $100 \mu \mathrm{M}$ & 44.04 & 5.87 & 0.00692 \\
\hline 2-(2-phenylethenyl)quinoline & $1 \mu \mathrm{M}$ & 3.77 & 0.68 & 0.0162 \\
\cline { 2 - 5 } & $10 \mu \mathrm{M}$ & 44.46 & 4.58 & 0.00368 \\
\cline { 2 - 5 } & $100 \mu \mathrm{M}$ & 258.30 & 6.76 & 0.000211 \\
\hline
\end{tabular}


Table S18. ER Antagonism Test Results

\begin{tabular}{|l|l|l|}
\hline Sample & $\begin{array}{l}\text { \% of } \\
\text { nM E2 }\end{array}$ & $\begin{array}{l}\text { Standard } \\
\text { deviation }\end{array}$ \\
\hline method blank & 112.03 & 7.83 \\
\hline E2 1 nM & 100.00 & 11.33 \\
\hline LRK1 & 118.69 & 14.48 \\
\hline LRK2 & 121.07 & 17.98 \\
\hline LRK3 & 118.98 & 6.61 \\
\hline MWS1 & 121.71 & 13.09 \\
\hline MWS2 & 125.17 & 4.42 \\
\hline MWS3 & 117.82 & 13.63 \\
\hline RLS1 & 118.04 & 15.00 \\
\hline RLS2 & 139.47 & 15.15 \\
\hline RLS3 & 73.46 & 2.30 \\
\hline TBS1 & 105.97 & 7.77 \\
\hline TBS2 & 108.22 & 14.22 \\
\hline TBS3 & 95.05 & 5.79 \\
\hline COF1 & 108.53 & 5.69 \\
\hline COF2 & 106.82 & 5.73 \\
\hline COF3 & 98.49 & 4.84 \\
\hline & & \\
\hline & \\
\hline
\end{tabular}


Table S19. AR Antagonism Test Results

\begin{tabular}{|l|l|l|}
\hline & $\begin{array}{l}\text { \% of 10 } \\
\text { uM T }\end{array}$ & $\begin{array}{l}\text { Standard } \\
\text { deviation }\end{array}$ \\
\hline method blank & 104.77 & 2.57 \\
\hline T 10 uM & 100.00 & 23.97 \\
\hline LRK1 & 96.49 & 7.19 \\
\hline LRK2 & 133.54 & 24.48 \\
\hline LRK3 & 297.93 & 23.01 \\
\hline MWS1 & 387.59 & 73.78 \\
\hline MWS2 & 311.42 & 5.27 \\
\hline MWS3 & 279.59 & 32.67 \\
\hline RLS1 & 327.25 & 14.84 \\
\hline RLS2 & 88.76 & 12.23 \\
\hline RLS3 & 26.08 & 1.76 \\
\hline TBS1 & 89.93 & 8.88 \\
\hline TBS2 & 267.45 & 17.17 \\
\hline TBS3 & 276.17 & 25.96 \\
\hline COF1 & 293.62 & 13.47 \\
\hline COF2 & 246.67 & 12.25 \\
\hline COF3 & 254.86 & 10.91 \\
\hline & & \\
\hline
\end{tabular}




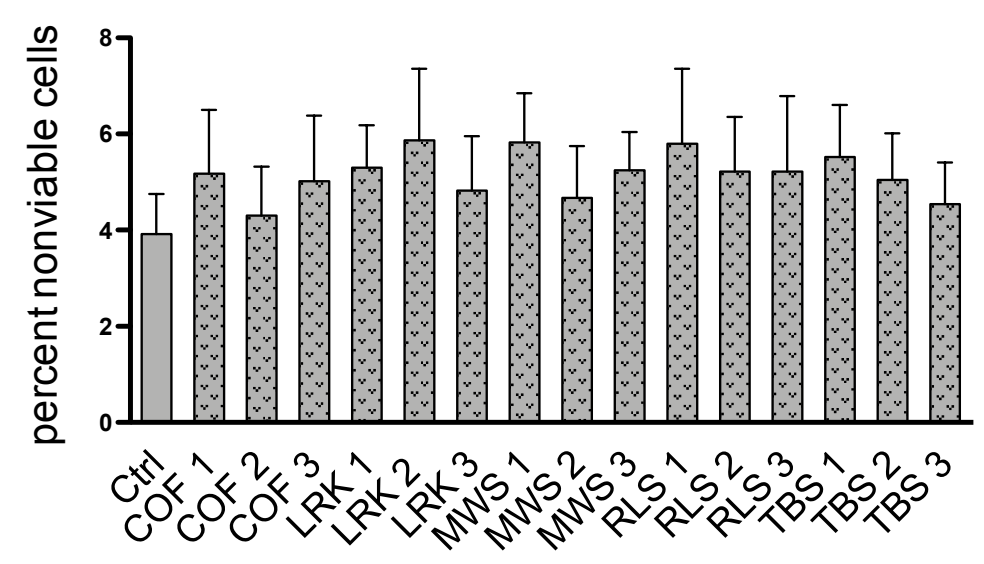

Figure S1. Effect of wildfire ash samples from Tubbs fire locations on cell viability. U937 derived macrophages were treated with extracts from ash samples for $24 \mathrm{~h}$ and cell viability was tested via trypan blue exclusion test. 


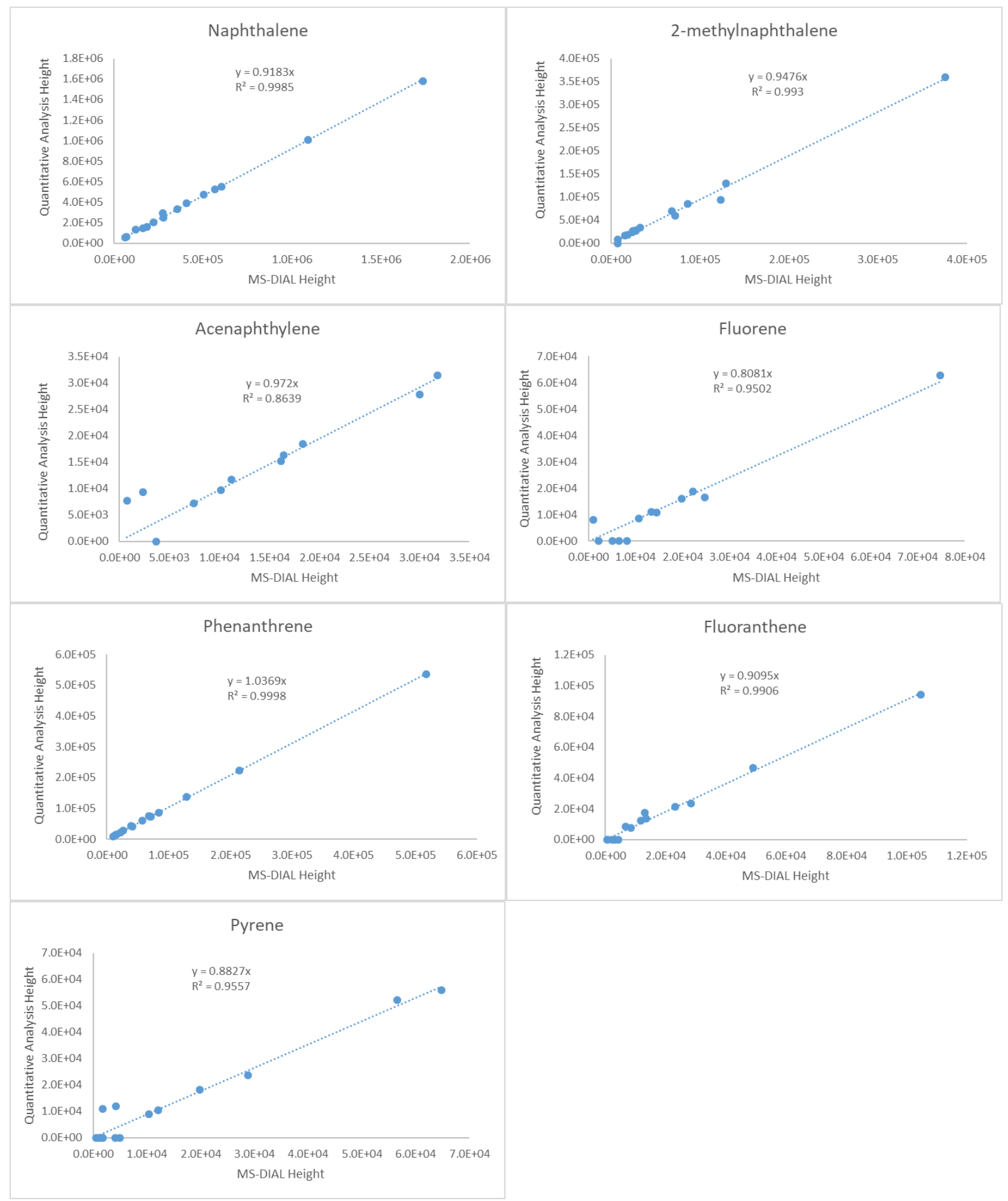

Figure S2. Comparison of peak heights for confirmed polycyclic aromatic hydrocarbon standards from Agilent Quantitative Analysis B.09 with the peak heights of the corresponding aligned and identified features from MS-DIAL 
Figure S3. Tentative identification, molecular structure, and mass spectra of GC-EI features listed in Table S14.

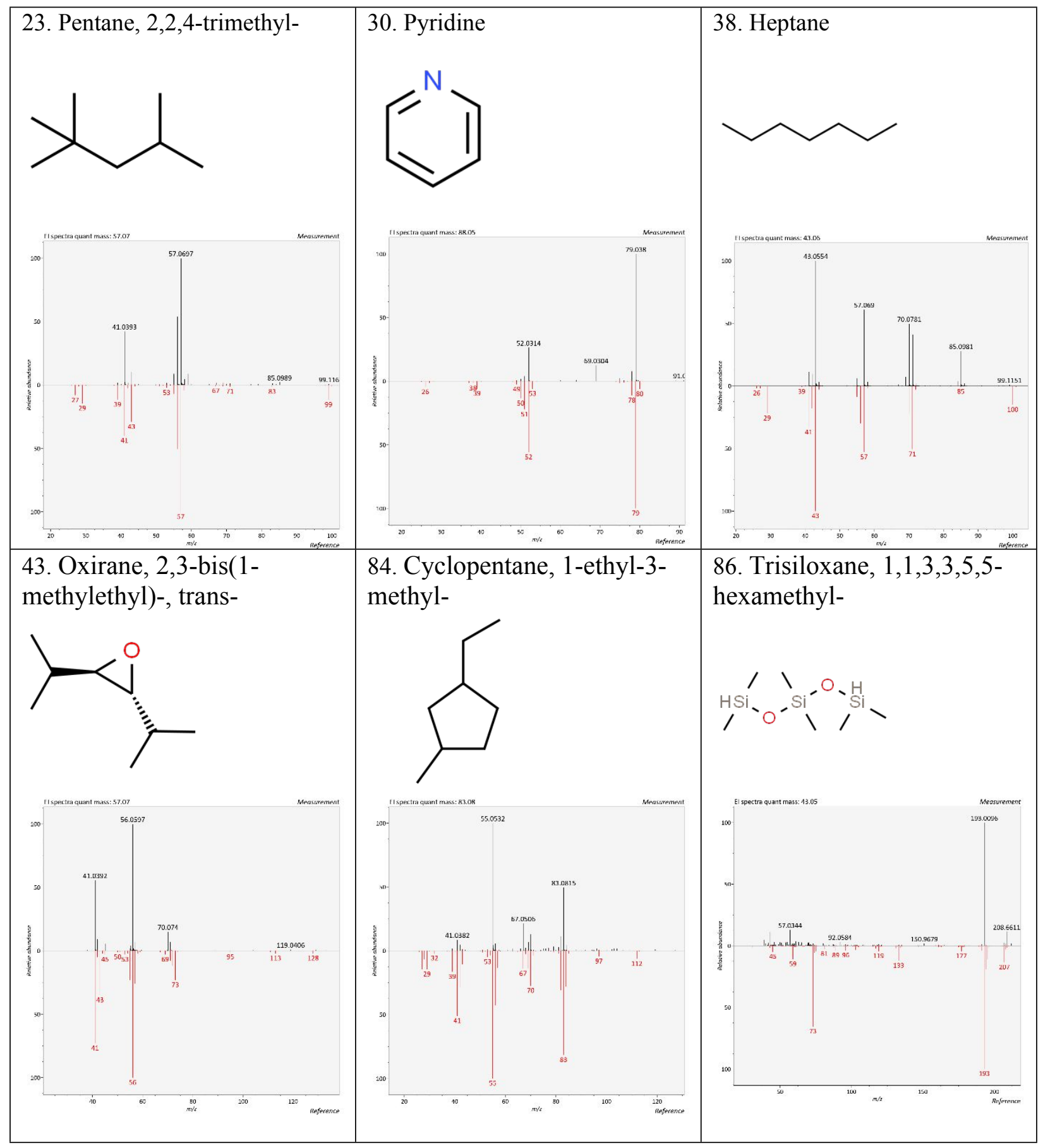




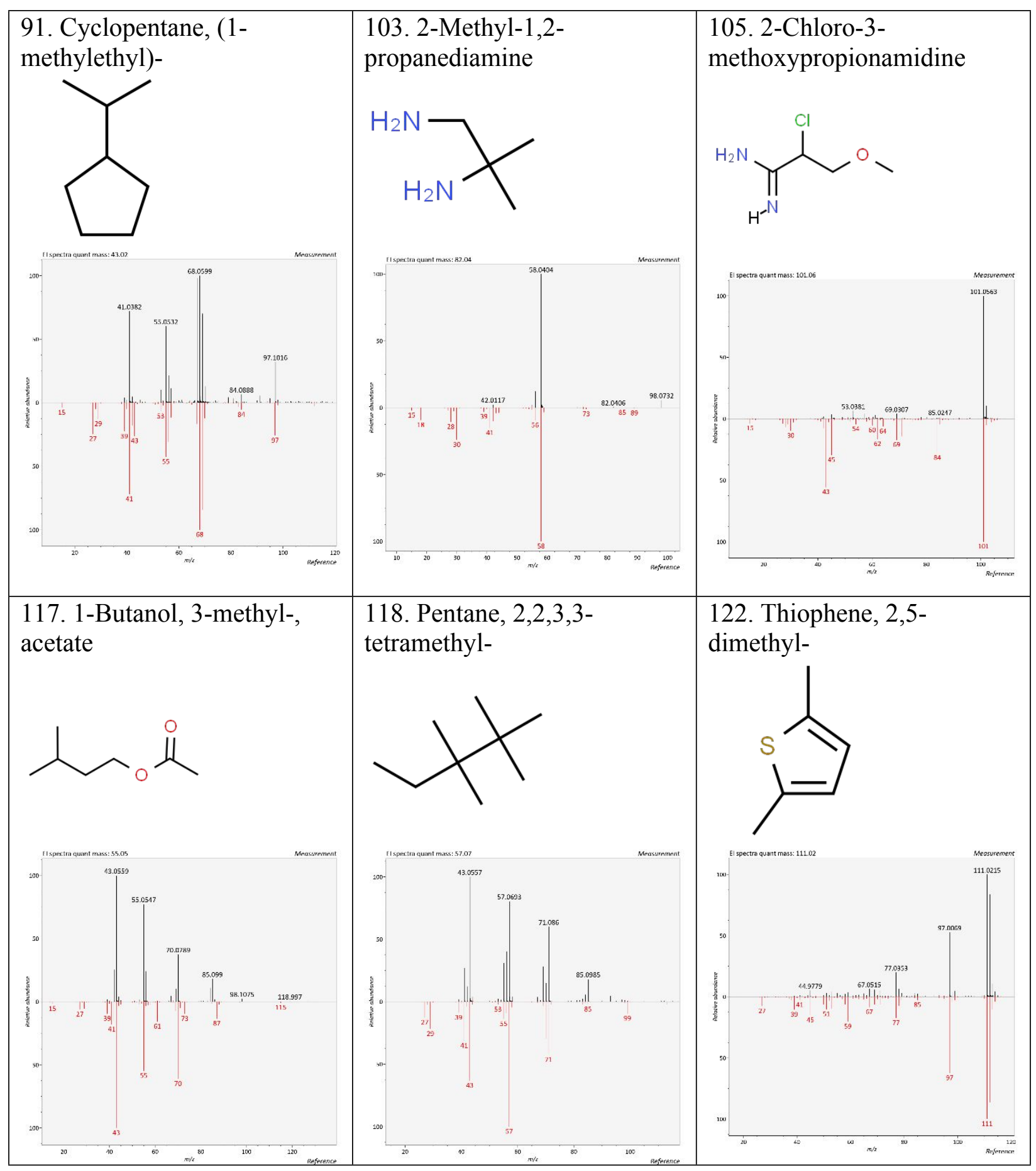




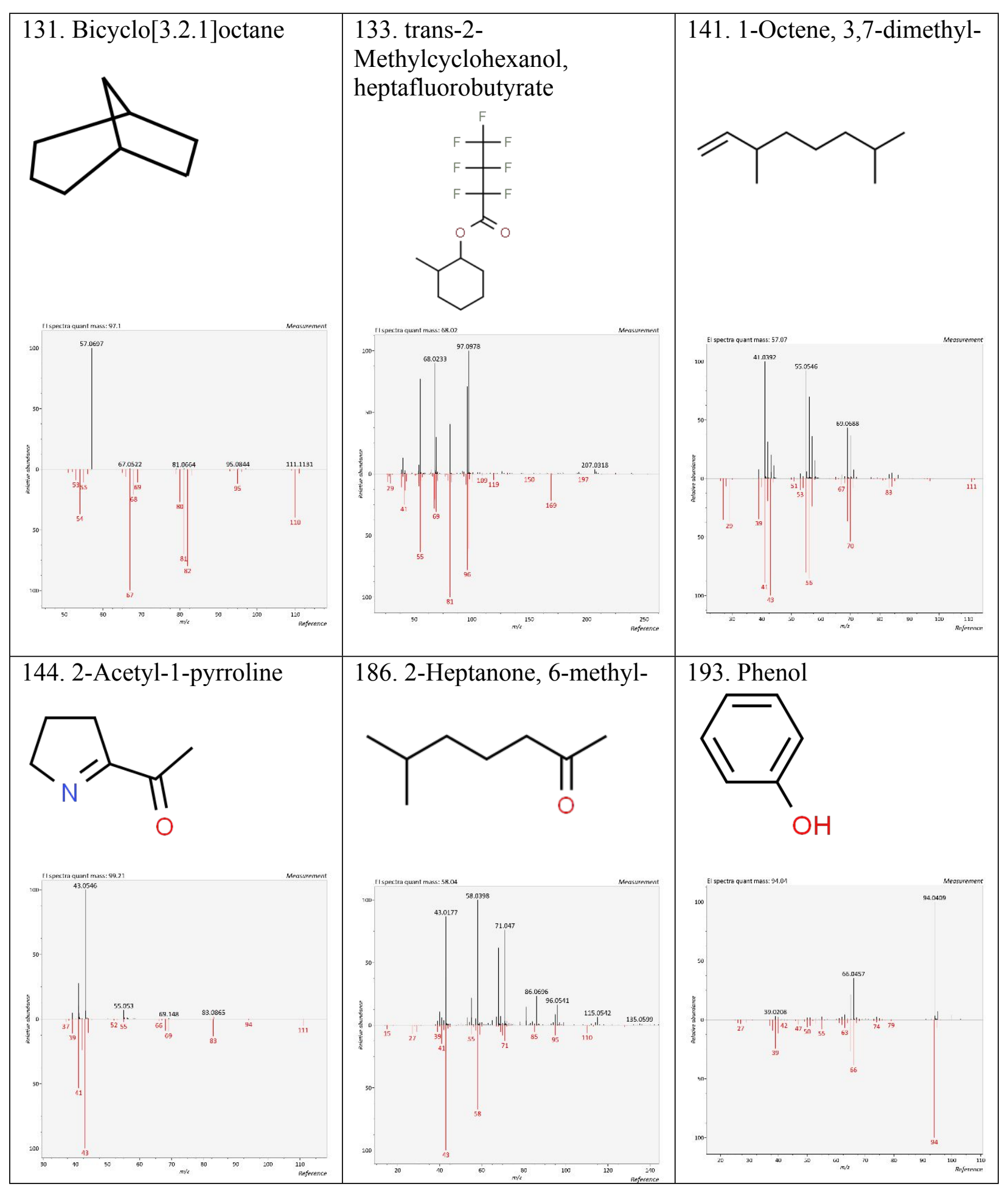




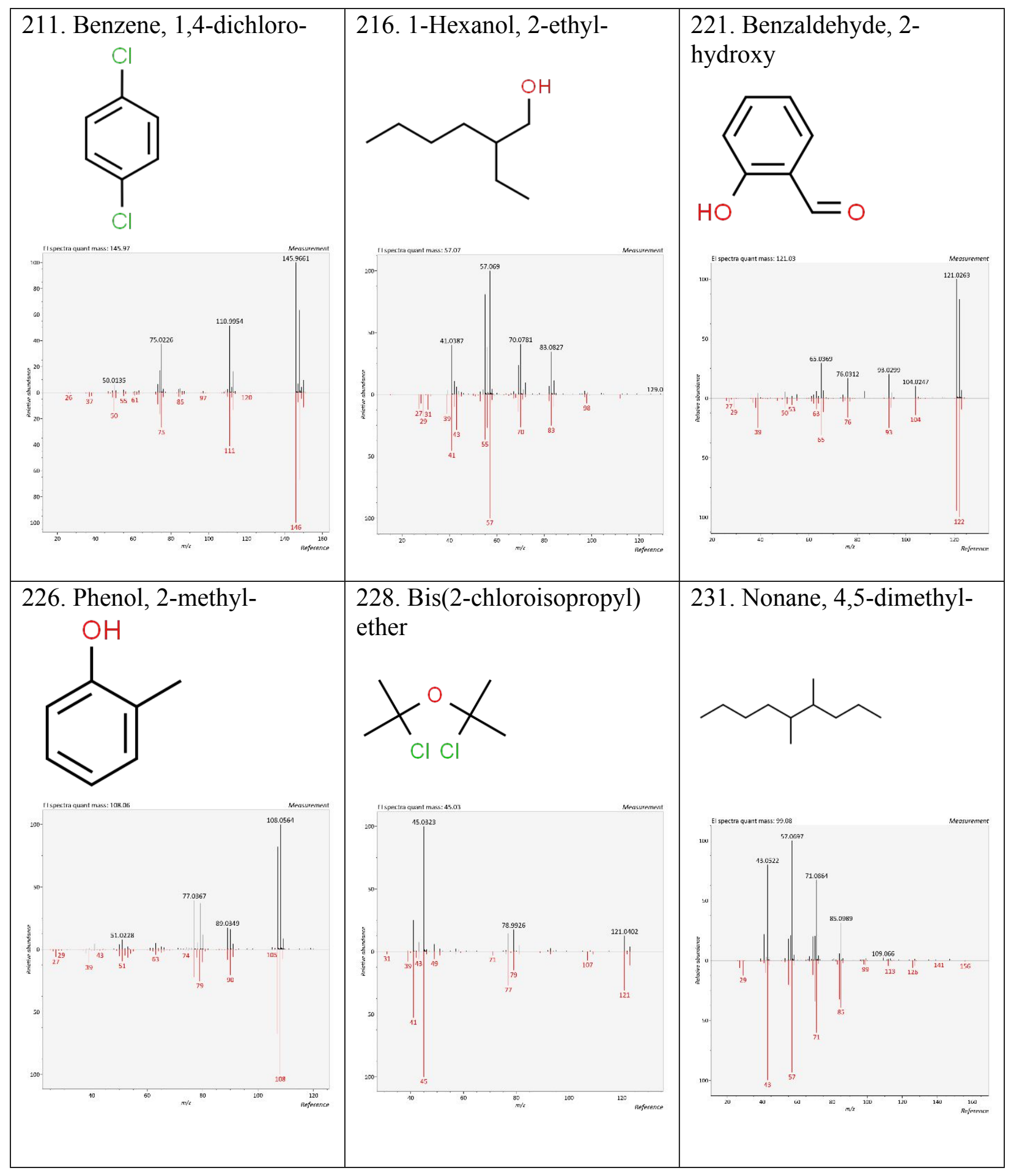




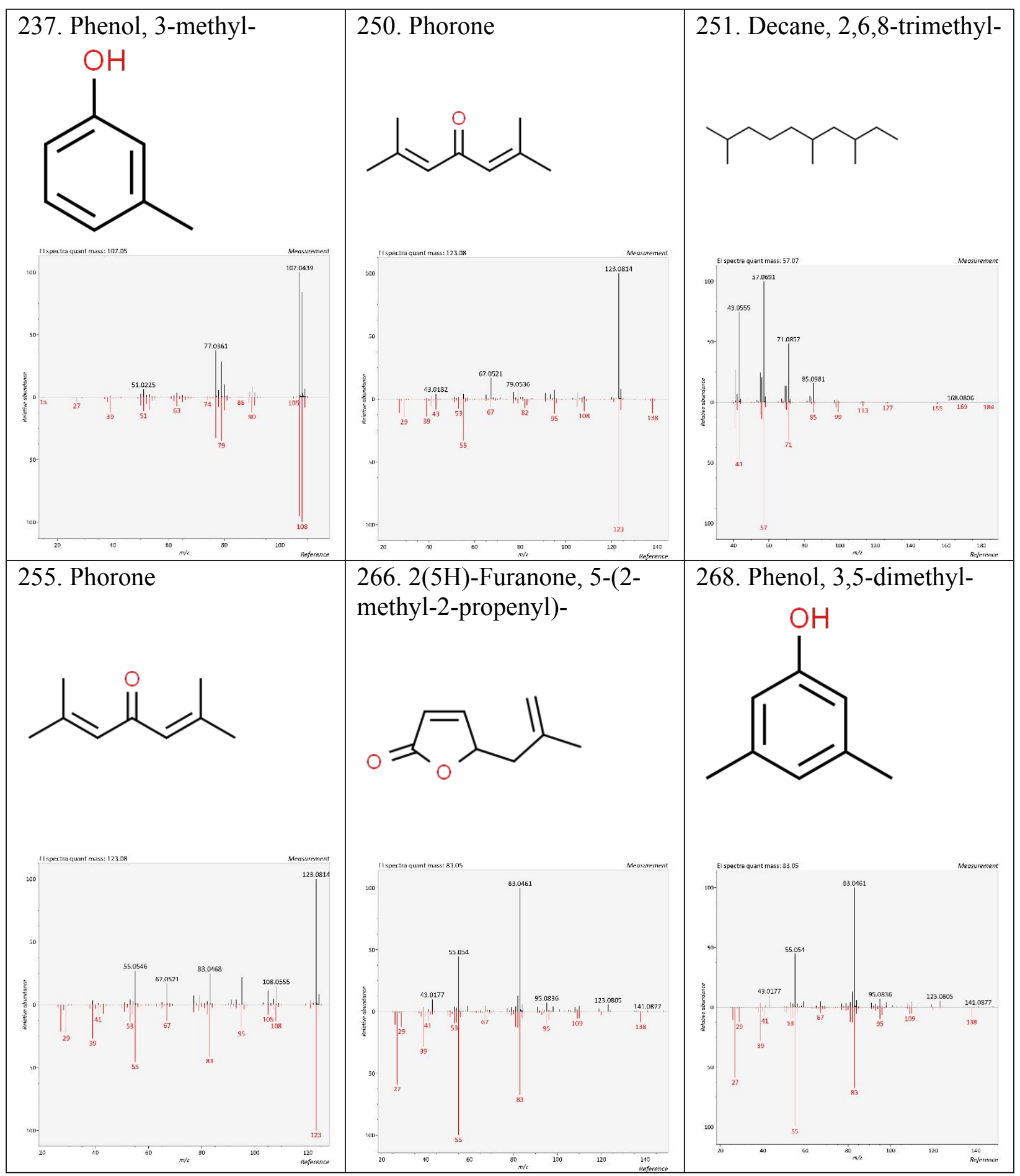




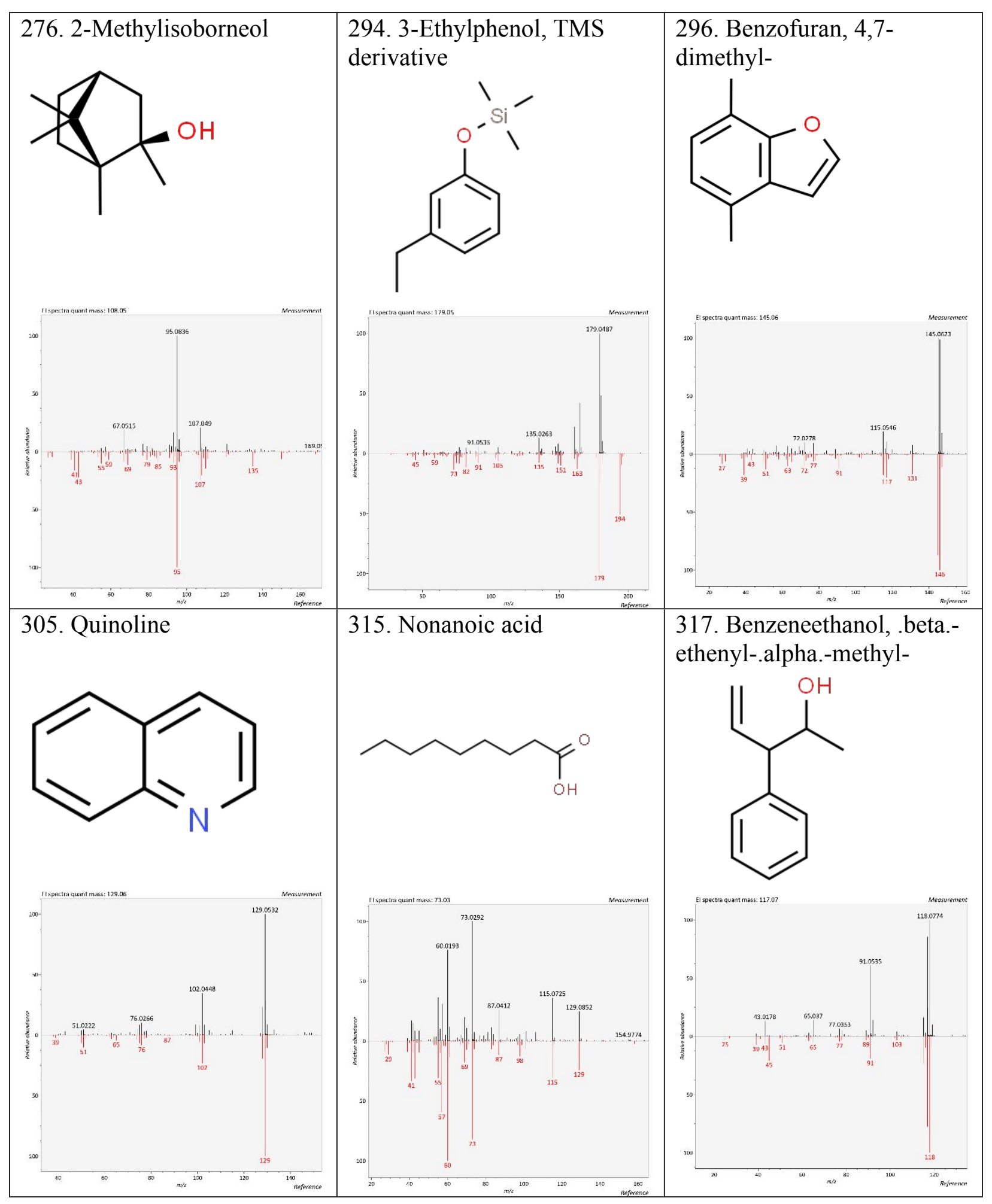




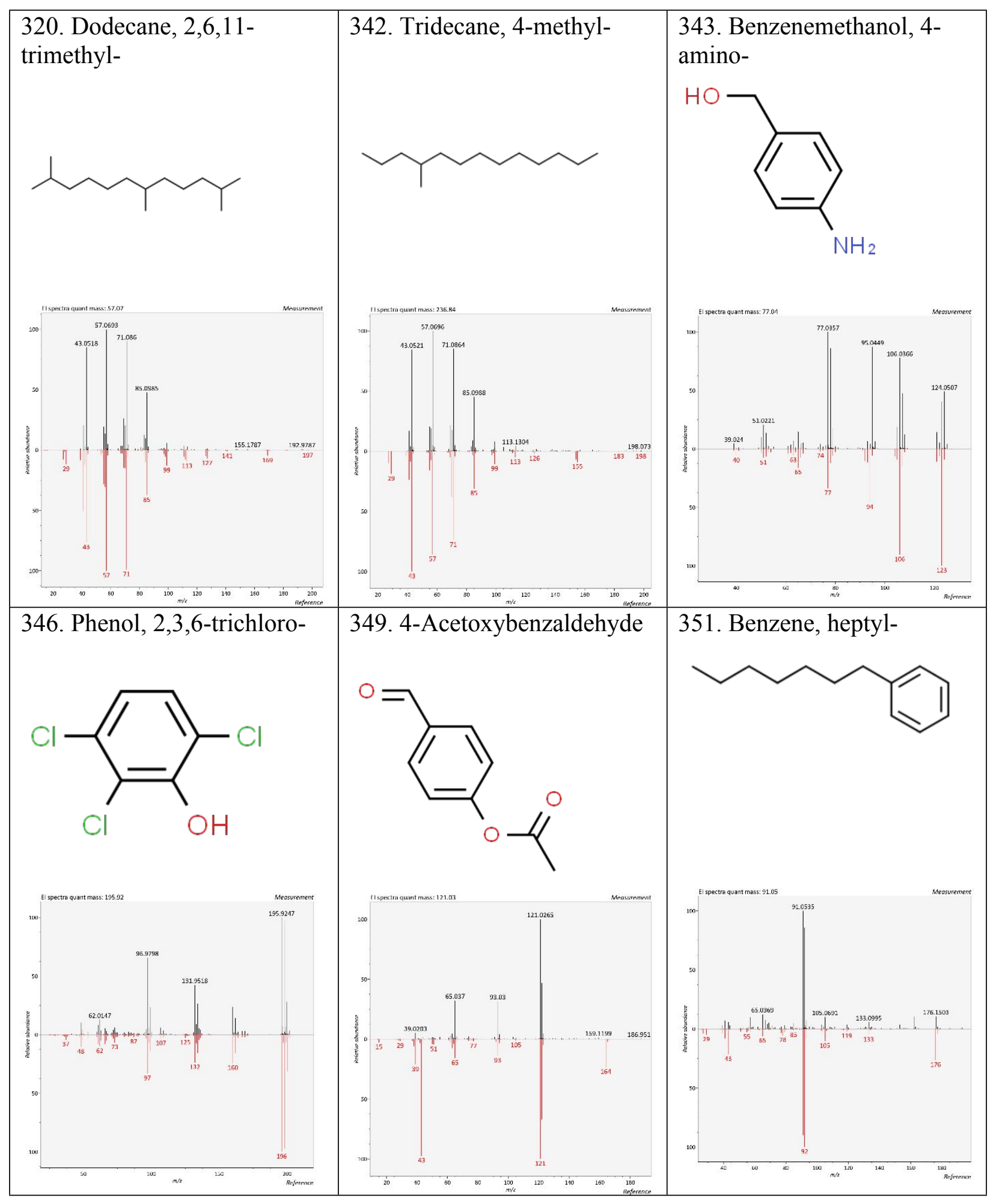




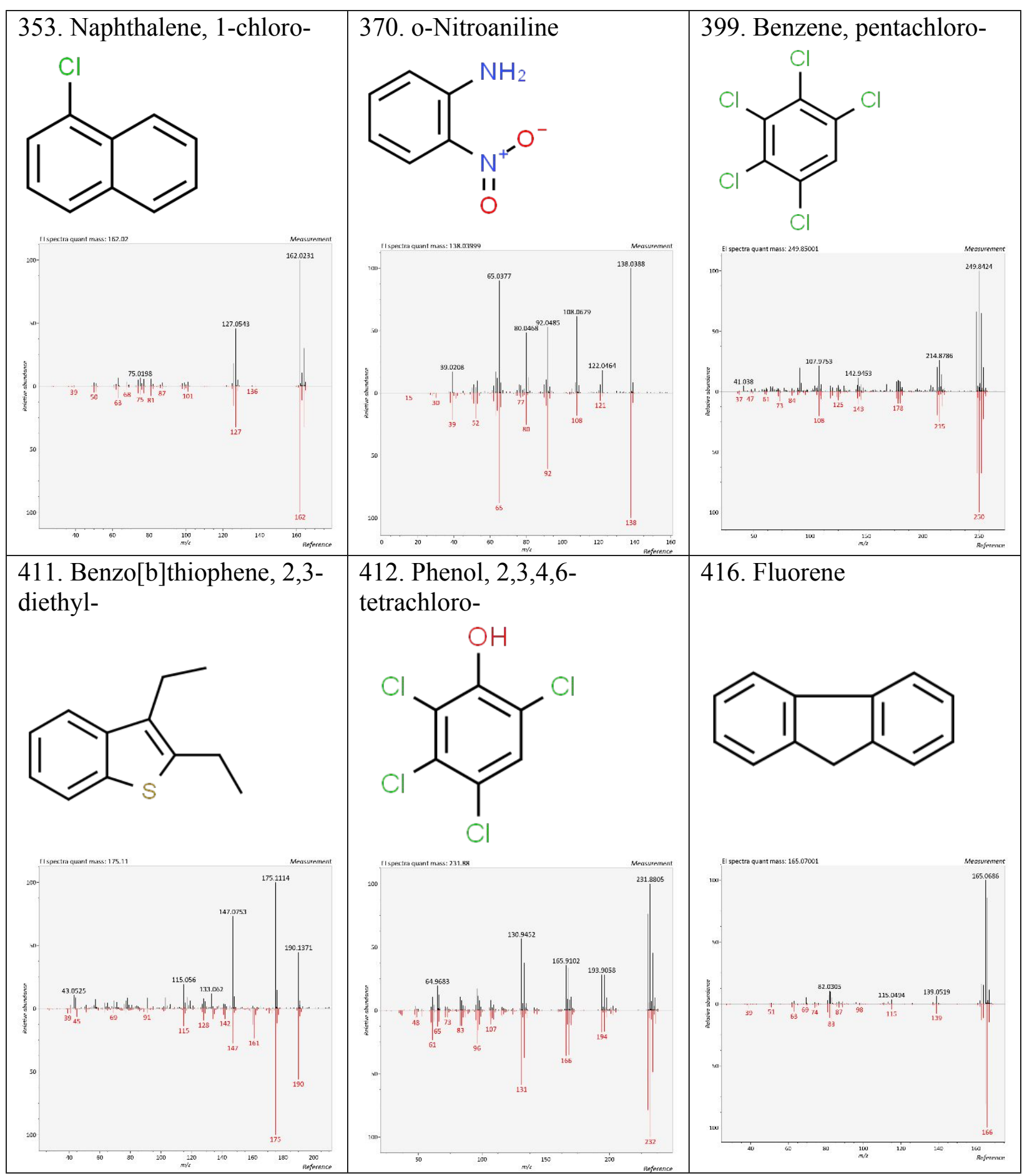




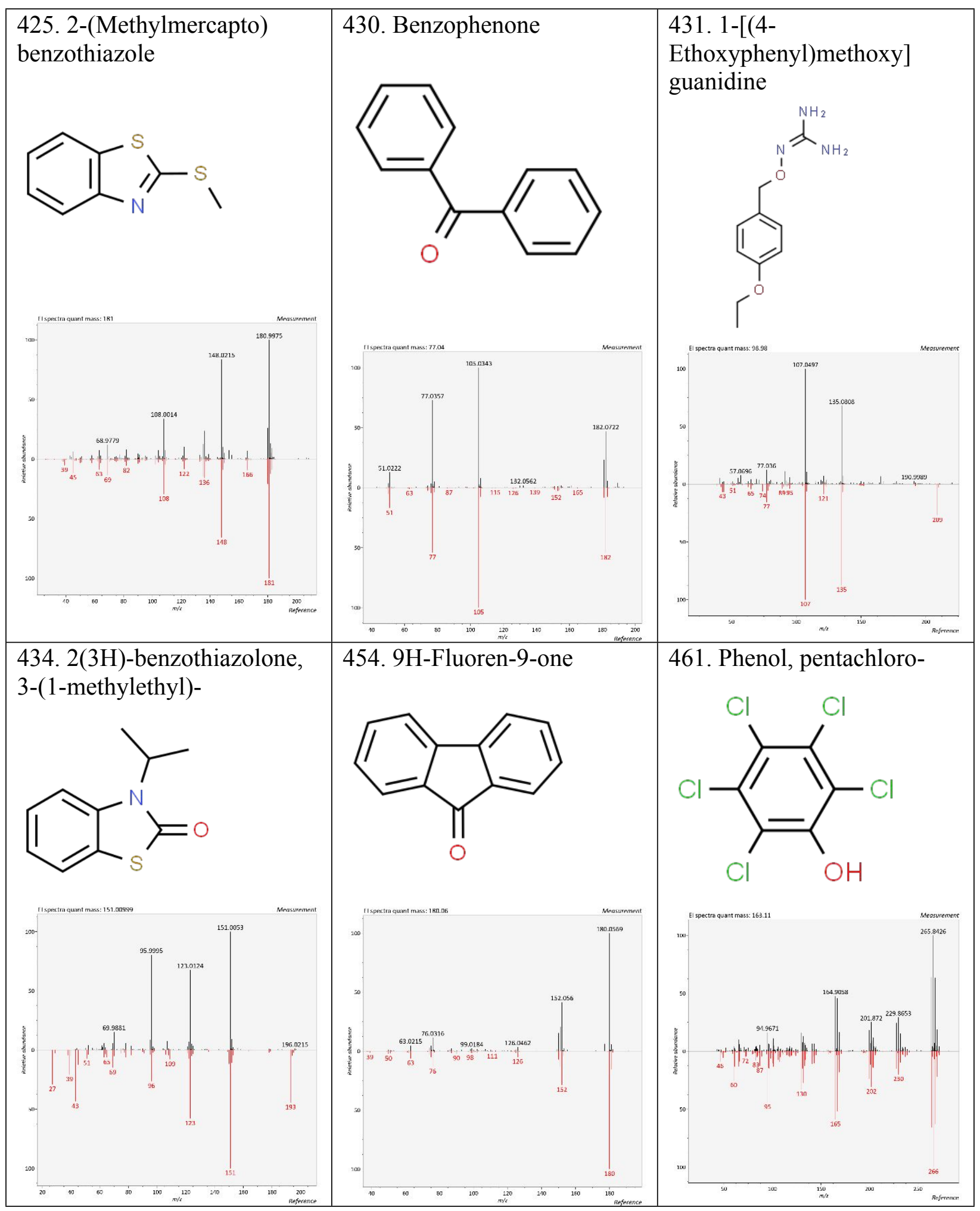




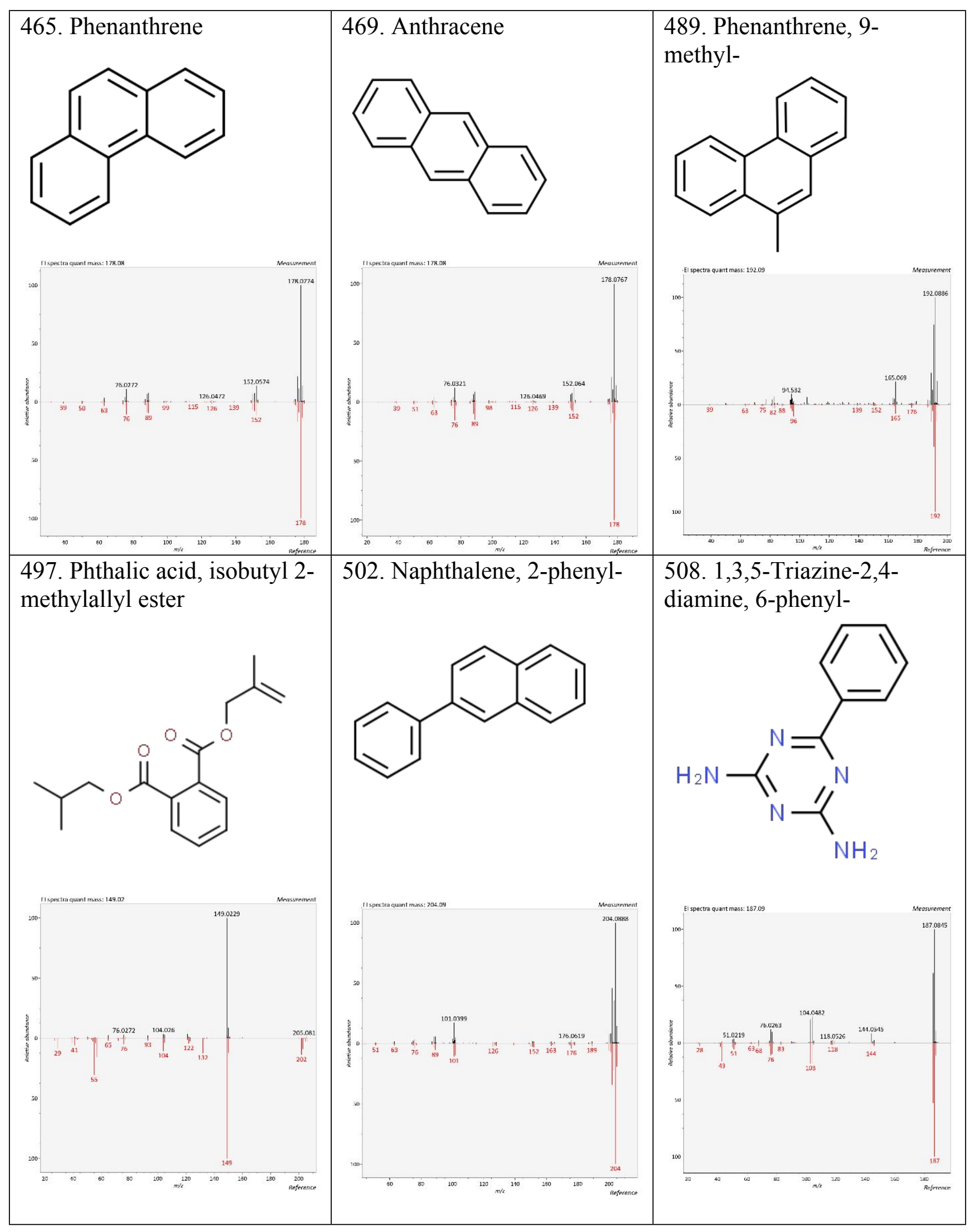




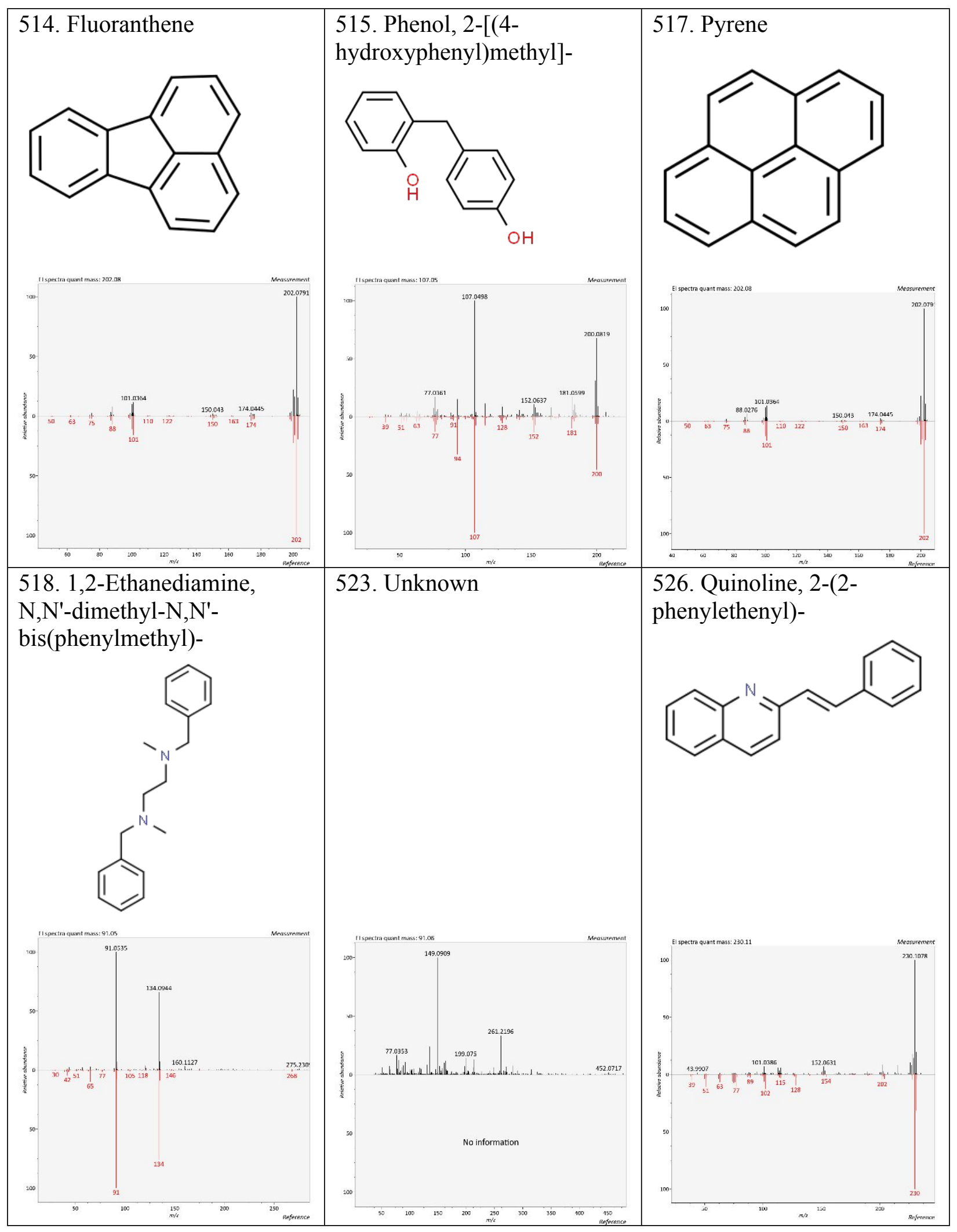




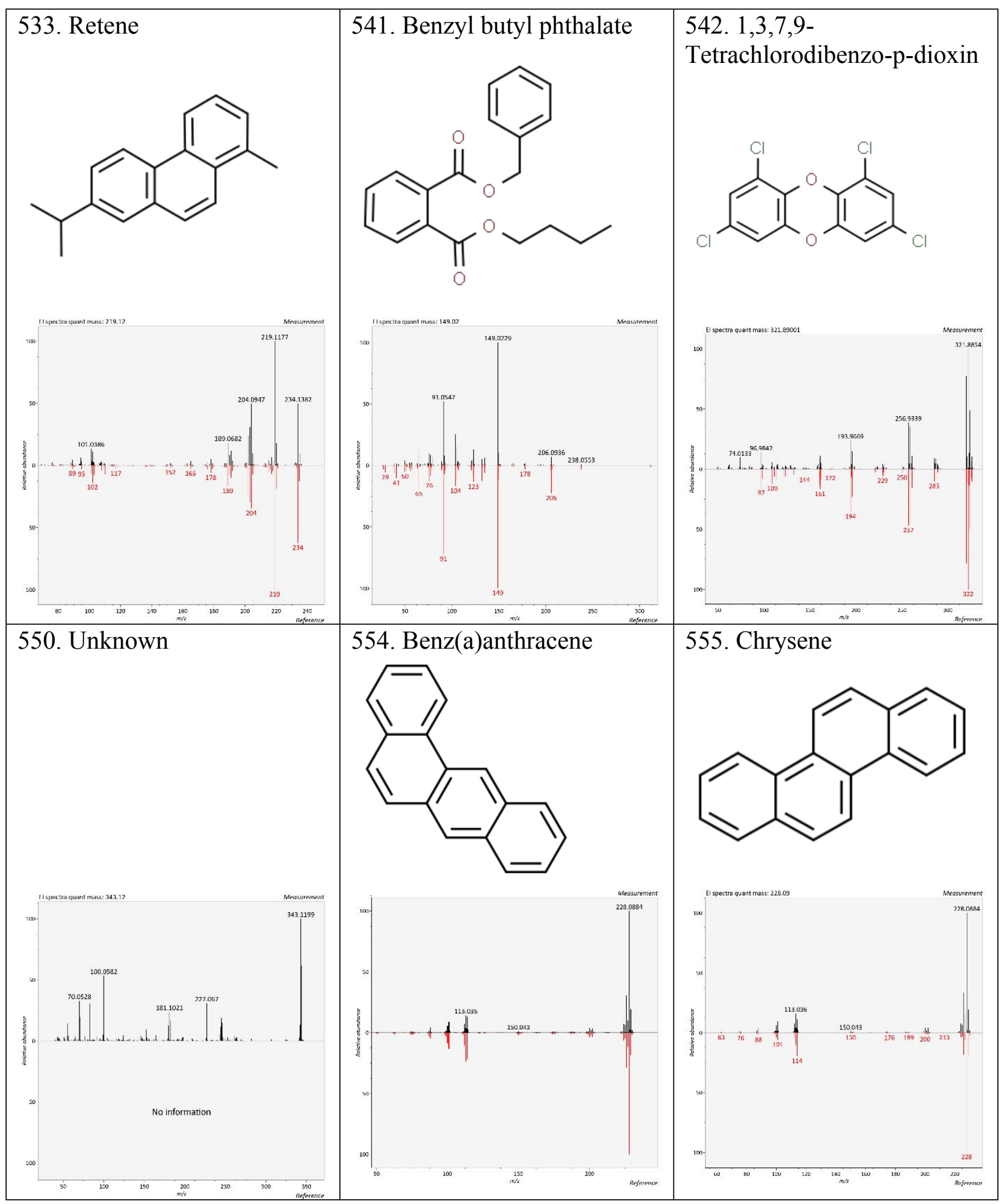




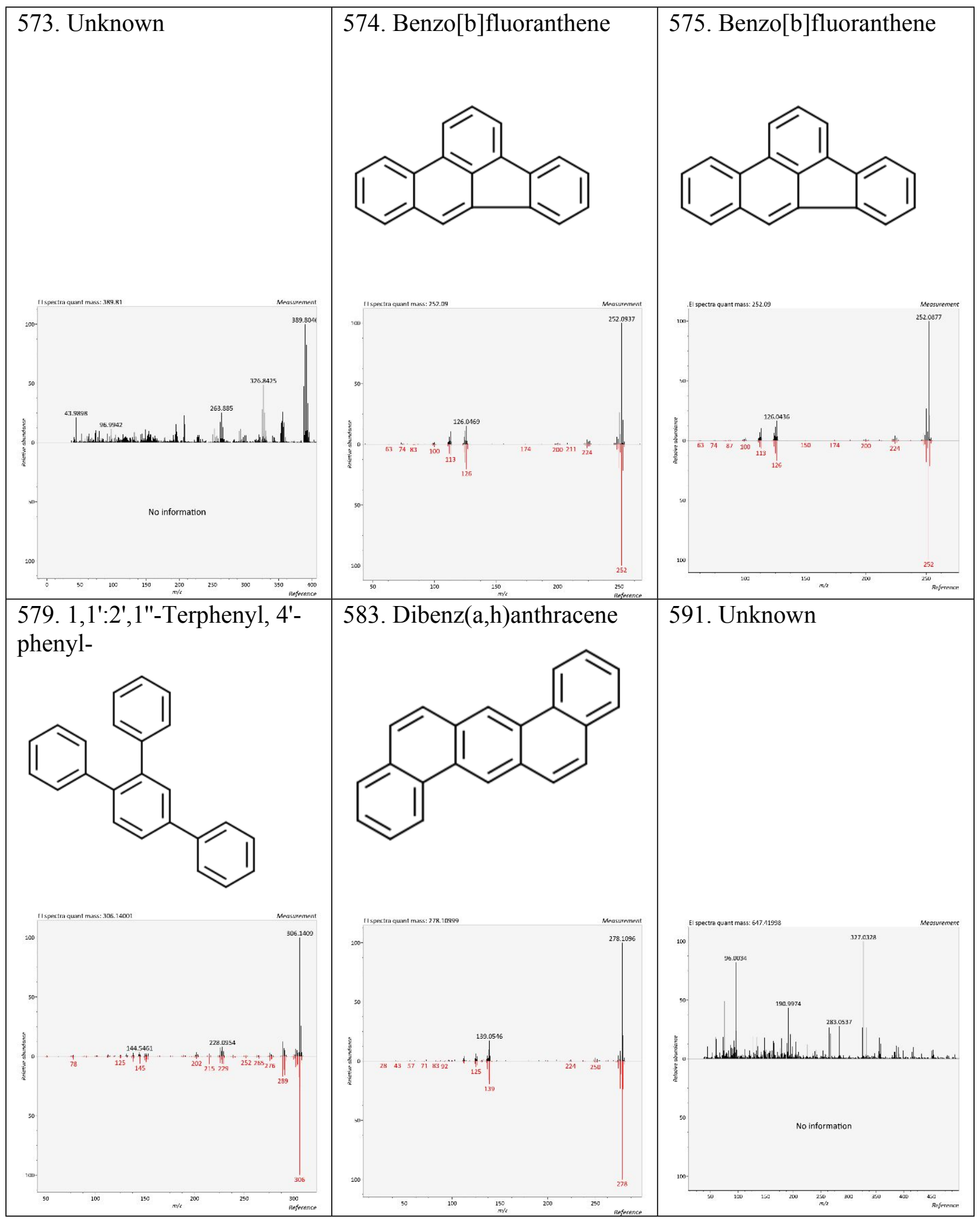


Figure S4. MS-FINDER annotations for selected LC-ESI+ features listed in Table S15 including proposed molecular structures and comparison of experimental targeted MS/MS spectra and in silico MS/MS spectra

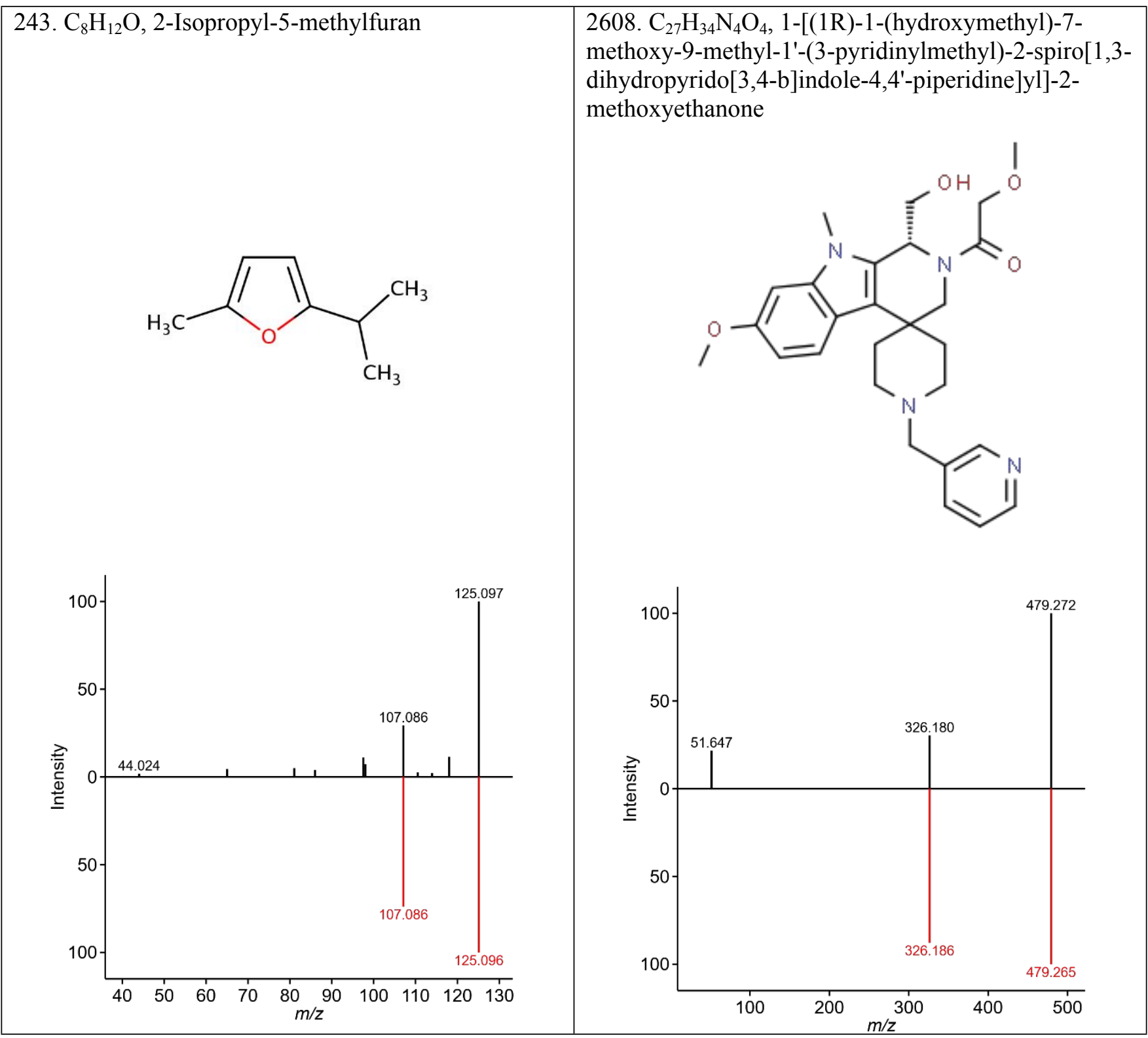


Figure S5. MS-FINDER annotations for selected LC-ESI- features listed in Table S16 including proposed molecular structures and comparison of experimental targeted MS/MS spectra and in silico MS/MS spectra

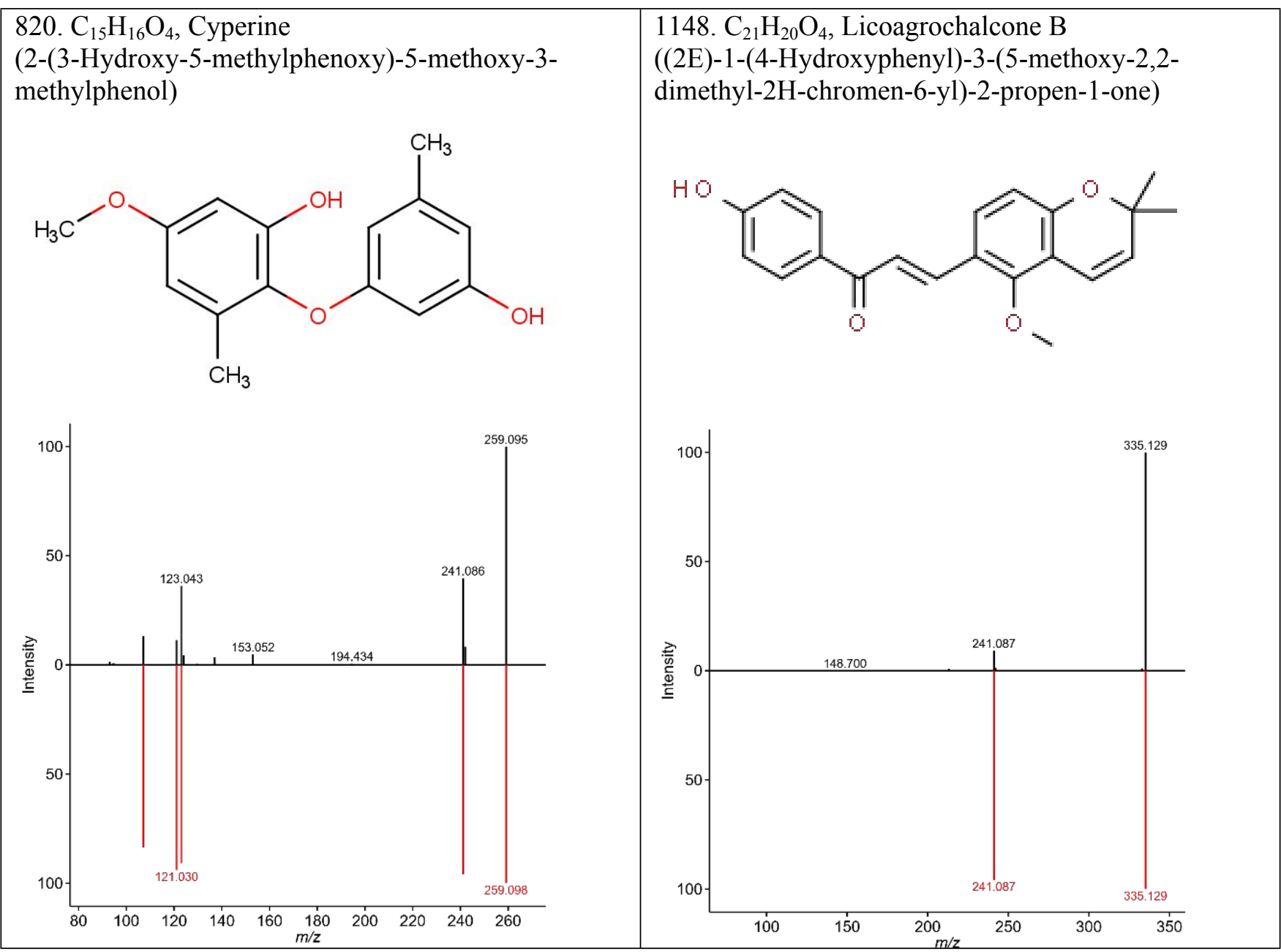

